\title{
REVISED Testing and tracking in the UK: A dynamic causal
}

\section{modelling study [version 2; peer review: 1 approved, 1}

\section{approved with reservations]}

\author{
Karl J. Friston (iD1, Thomas Parr (D1), Peter Zeidman1, Adeel Razi (D1,2, \\ Guillaume Flandin1, Jean Daunizeau3, Oliver J. Hulme ${ }^{4,5}$, Alexander J. Billig(1D6, \\ Vladimir Litvak¹, Cathy J. Price1, Rosalyn J. Moran (iD7, Christian Lambert (iD)1 \\ ${ }^{1}$ The Wellcome Centre for Human Neuroimaging, University College London, London, WC1N 3BG, UK \\ ${ }^{2}$ Turner Institute for Brain and Mental Health, Monash University, Clayton, VIC, 3800, Australia \\ ${ }^{3}$ Institut du Cerveau et de la Moelle épinière, INSERM UMRS, Paris, France \\ ${ }^{4}$ Danish Research Centre for Magnetic Resonance, Centre for Functional and Diagnostic Imaging and Research, Copenhagen \\ University Hospital Hvidovre, Hvidovre, Denmark \\ ${ }^{5}$ London Mathematical Laboratory, London, UK \\ ${ }^{6}$ Ear Institute, University College London, London, UK \\ ${ }^{7}$ Centre for Neuroimaging Science, Department of Neuroimaging, IoPPN, King's College London SE1 1UL, London, UK
}

\author{
V2 First published: 17 Jun 2020, 5:144 \\ https://doi.org/10.12688/wellcomeopenres.16004.1 \\ Latest published: 15 Feb 2021, 5:144 \\ https://doi.org/10.12688/wellcomeopenres.16004.2
}

\section{Abstract}

By equipping a previously reported dynamic causal modelling of COVID-19 with an isolation state, we were able to model the effects of self-isolation consequent on testing and tracking. Specifically, we included a quarantine or isolation state occupied by people who believe they might be infected but are asymptomatic-and could only leave if they test negative. We recovered maximum posteriori estimates of the model parameters using time series of new cases, daily deaths, and tests for the UK. These parameters were used to simulate the trajectory of the outbreak in the UK over an 18-month period. Several clear-cut conclusions emerged from these simulations. For example, under plausible (graded) relaxations of social distancing, a rebound of infections is highly unlikely. The emergence of a second wave depends almost exclusively on the rate at which we lose immunity, inherited from the first wave. There exists no testing strategy that can attenuate mortality rates, other than by deferring or delaying a second wave. A testing and tracking policy-implemented at the present time-will defer any second wave beyond a time horizon of 18 months. Crucially, this deferment is within current testing capabilities (requiring an efficacy of tracing and tracking of about $20 \%$ of asymptomatic infected cases, with 50,000 tests per day). These conclusions are based upon a dynamic causal model for which we provide some construct and face validation-using a comparative analysis of the

\section{Open Peer Review}

\begin{tabular}{|c|c|c|}
\hline \multicolumn{3}{|c|}{ Approval Status ? } \\
\hline & 1 & 2 \\
\hline \multicolumn{3}{|l|}{ version 2} \\
\hline (revision) & & $\checkmark$ \\
\hline 15 Feb 2021 & & view \\
\hline version 1 & $?$ & X \\
\hline 17 Jun 2020 & view & view \\
\hline
\end{tabular}

1. Marta Colaneri iD, Fondazione IRCCS

Policlinico San Matteo, Pavia, Italy

2. Lin Wang ID, University of Cambridge,

Cambridge, UK

Any reports and responses or comments on the article can be found at the end of the article. 
United Kingdom and Germany, supplemented with recent serological

studies.

Keywords

coronavirus, epidemiology, compartmental models, dynamic causal

modelling, variational, Bayesian

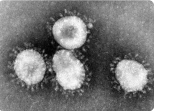

This article is included in the Coronavirus

(COVID-19) collection.

Corresponding author: Adeel Razi (adeel.razi@monash.edu)

Author roles: Friston KJ: Conceptualization, Data Curation, Formal Analysis, Funding Acquisition, Investigation, Methodology, Resources, Software, Validation, Visualization, Writing - Original Draft Preparation; Parr T: Methodology, Writing - Review \& Editing; Zeidman P: Methodology, Software, Validation, Writing - Review \& Editing; Razi A: Methodology, Software, Validation, Writing - Review \& Editing; Flandin G: Methodology, Software, Validation, Writing - Review \& Editing; Daunizeau J: Methodology, Writing - Review \& Editing; Hulme OJ: Methodology, Software, Validation; Billig AJ: Methodology, Writing - Review \& Editing; Litvak V: Methodology, Writing - Review \& Editing; Price CJ: Writing - Review \& Editing; Moran RJ: Methodology, Writing - Review \& Editing; Lambert C: Methodology, Writing - Review \& Editing

Competing interests: No competing interests were disclosed.

Grant information: This work was supported by Wellcome through core funding to the Wellcome Centre for Human Neuroimaging, UCL Queen Square Institute of Neurology [203147]; and a Principle Research Fellowship grant to KJF [088130]. AJB is supported by a Wellcome Trust grant [091681]. AR is funded by the Australian Research Council [Refs: DE170100128 and DP200100757]. CL is supported by an MRC Clinician Scientist award [MR/R006504/1].

The funders had no role in study design, data collection and analysis, decision to publish, or preparation of the manuscript.

Copyright: ( 2021 Friston $\mathrm{KJ}$ et al. This is an open access article distributed under the terms of the Creative Commons Attribution License, which permits unrestricted use, distribution, and reproduction in any medium, provided the original work is properly cited.

How to cite this article: Friston $\mathrm{KJ}$, Parr T, Zeidman P et al. Testing and tracking in the UK: A dynamic causal modelling study [version 2; peer review: 1 approved, 1 approved with reservations] Wellcome Open Research 2021, 5:144 https://doi.org/10.12688/wellcomeopenres.16004.2

First published: 17 Jun 2020, 5:144 https://doi.org/10.12688/wellcomeopenres.16004.1 


\section{REVISED Amendments from Version 1}

This updated version includes: i) some clarifications on technical aspects, and ii) limitations of the study are highlighted based on reviewers' comments and are listed in the Conclusion section.

Any further responses from the reviewers can be found at the end of the article

\section{Introduction}

This is the third in a series of technical reports that use dynamic causal modelling to explain and predict the current outbreak of COVID-19. The first report described an enhanced compartmental model based upon a factorisation of latent or hidden states generating timeseries data, such as new cases and daily deaths (Friston et al., 2020a). This model was concerned with an outbreak in a single region parameterised with an effective population size. The second report assembled several models of a single region, coupled by population flux between regions, to model the pandemic in the united states of America (Friston et al., 2020b). The focus of this multi-region model was on the genesis of second waves and a key, mechanistic, distinction between rebounds due to premature relaxation of social distancing and second waves due to loss of immunity. The basic conclusions of this second report were that a devolved social distancing strategy - that was sensitive to local metricspredicted better outcomes than a national or federal strategy. In this report, we return to the model of a single region or country and look more closely at strategies in terms of surveillance; specifically, the role of testing, tracing, and tracking.

The efficacy of contact tracing programs are now the focus of several modelling initiatives (Aleta et al., 2020; Ferretti et al., 2020; Giordano et al., 2020; Gurdasani \& Ziauddeen, 2020; Hellewell et al., 2020; Keeling et al., 2020), whose conclusions depend upon the form of the models used. Models that include social distancing and isolation of infected contacts suggest that a 'find', 'track', 'trace' and 'isolate' (FTTI) policy can ameliorate morbidity (Giordano et al., 2020; Kretzschmar et al., 2020).

To address the efficacy of FTTI, we equipped the dynamic causal model (DCM) with a further location state; namely, a state of self-isolation or quarantine. People entered the state when experiencing symptoms or awaiting a PCR test. They remained isolated for seven days unless they received news that the test was negative. This construction accommodates the mechanistic process by which FTTI operates. In other words, the agenda behind testing and tracking is to isolate those people who are infected before they become contagious. This allows one to move back in time and pre-empt the reproduction of the virus in the population. However, to do this, it is necessary to identify people who are asymptomatic, thereby enriching or enhancing the probability that targeted testing will identify infected individuals. We operationalise this testing and tracking strategy in terms of its efficacy. Here, efficacy is defined as the probability that I will be offered a test by a programme of test and tracking if I am infected and asymptomatic. Under this parameterisation, an ineffective testing and tracking renders this probability zero. Conversely, and efficiency of $100 \%$ means that if I am infected and asymptomatic, I will certainly be tested. Clearly, for a large population, high levels of efficacy may not be attainable; however, lower levels may be sufficient to either suppress the reproduction rate of viral transmission (Aleta et al., 2020) or defer the emergence of any second wave until an efficacious programme of vaccination is in place (or effective treatments have been established).

To model different aspects of testing and surveillance, we had to carefully parameterise testing along a number of dimensions. To do this, we assume that there was a small, time-dependent probability of being tested on any given day. This testing probability was modelled in terms of a constant baseline, a testing component sensitive to the prevalence of infection in the population and a sustained component following the first wave. This sustained component was modelled as proportional to the level of herd immunity acquired after successive waves of infection. Having parameterised the testing probability, the selectivity of testing was parameterised in terms of the probability of being tested if infected, relative to not being infected. Finally, if I am infected but asymptomatic, then the probability of being tested is supplemented with a test and track componentthat could start at the beginning of the outbreak, or any subsequent time. This may sound a rather involved parameterisation; however, it is a minimal model needed to generate the number of positive cases reported, given the latent prevalence of infection. This follows because the number of positive cases depends not only on the probability of being tested but whether I am more likely to be tested if I am infected (e.g., I work in a care home) or not (e.g., I have been selected at random by a screening survey).

Please see Figure 1 and Table 1 for a brief review of the model (and the Methods for the parameterisation of self-isolation and testing). With this model and its parameters in place, one can now fit the model to empirical data until the present day. Crucially, because the parameters of this model do not change, they can then be used to forecast the future trajectory, under various adjustments to the testing parameters. The following conclusions foreshadow the results of these simulations:

- There is no plausible parameterisation of the model that would or could permit a flareup or rebound of the outbreak following a relaxation of social distancing measures. This is under the qualified assumption that social distancing continues to be operating in the way it is modelled-and inferred-on the basis of the empirical evidence to date. In short, provided there is a graded, parametric response to the prevalence of infection in the population, there will be no rebound in the weeks following the peak of the first wave.

- A second wave is inevitable. The timing of the second wave depends almost exclusively on the rate at which immunity is lost. In other words, under the assumption that being infected confers immunity-and that the immunity lasts for a given period - the period of immunity determines the timing of the second wave. This second wave is 


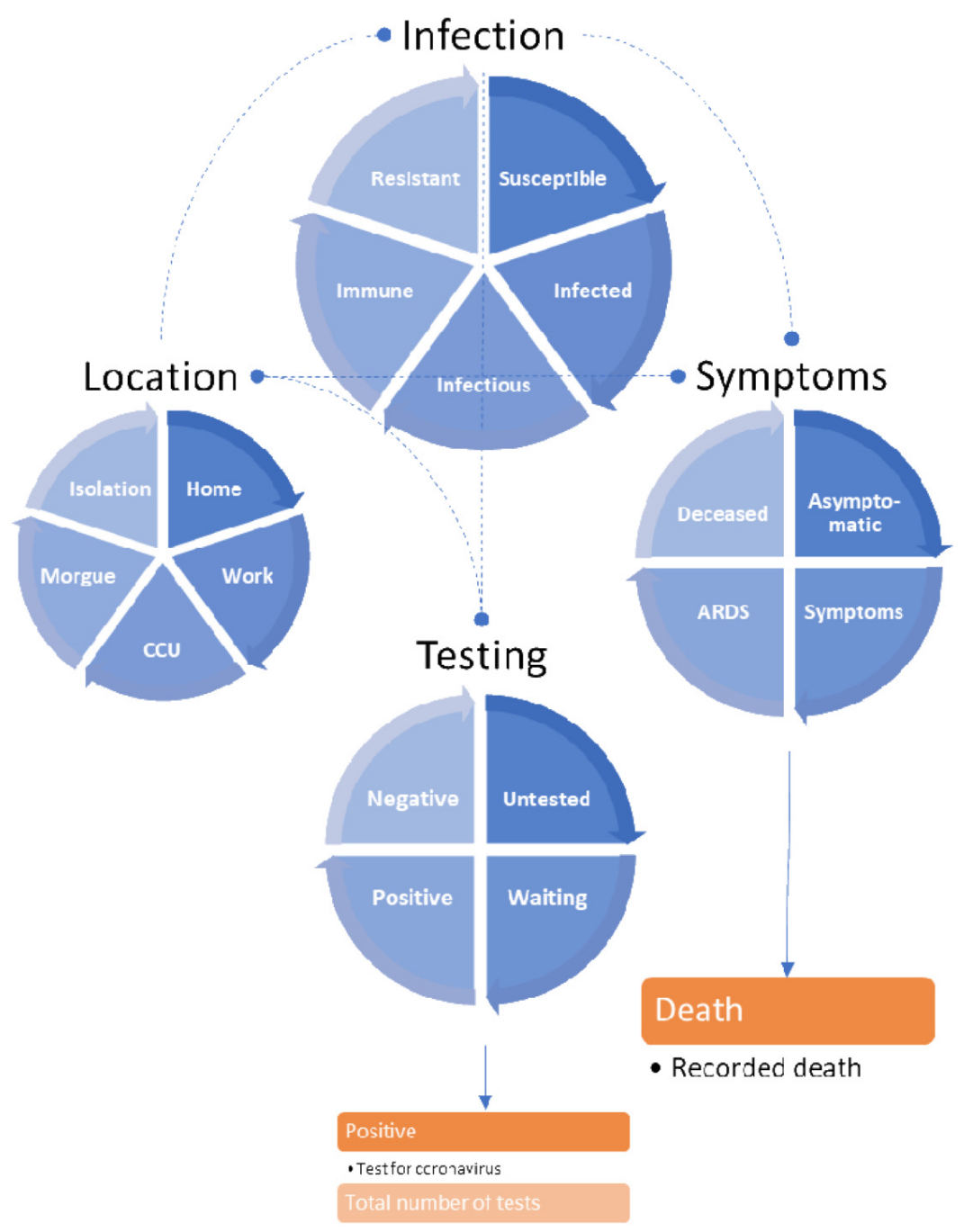

Figure 1. The LIST model. This schematic summarises the LIST (location, infection symptom and testing) generative model used for the following simulations. This model is formally similar to that described in (Friston et al., 2020b). Here, it has been augmented with an extra location state (isolation) to model people who are self-isolating because they think they may be infectious. Note that in this model there are no absorbing states. In other words, one can leave any state within any of the four factors. For example, one only occupies the state of being deceased (or testing positive and negative) for a day and then moves to asymptomatic (or untested) on the following day. This ensures that the total population is conserved, i.e., probability mass is conserved in terms of the ensemble density. Furthermore, it enables the occupancy of various states to be interpreted in terms of the rate of daily expression. The blue boxes correspond to states or compartments. The states within any factor are mutually exclusive, where the factors embody the factorial form of this compartmental model. In other words, every individual in the population has to be in one of several possible states that are characterised in terms of four factors or attributes. The orange boxes represent the outputs that are generated by this dynamic causal model, in this instance, daily reports of positive tests, daily tests and deaths.

mechanistically distinct from a fluctuation of, or rebound from, the first wave.

- There is no social distancing or surveillance strategy that will have any material impact on the total number of deaths accumulated from the onset of an outbreak to an idealised endemic equilibrium. However, certain strategies can defer waves of infection. Specifically, testing and tracking can defer expression of the second wave beyond a time horizon, after which vaccination or other therapeutic interventions will render it innocuous. In short, the mechanism by which strategic interventions operate is not by eliminating the infection but by slowing it down sufficiently, so that its pathogenicity is dissolved by viral mutation, vaccination, or therapeutic advances. Here, we assume a time horizon of 18 months.

- The most efficacious strategy for deferring a second wave is testing and tracking. Furthermore, the logistic requirements are within current capabilities. The same is 
Table 1. Parameters of the epidemic (LIST) model and priors, $\boldsymbol{N}(\boldsymbol{\eta}, \boldsymbol{C})$. (NB: prior means are for scale parameters $\theta=\exp (\vartheta)$ ).

\begin{tabular}{|c|c|c|c|c|}
\hline Number & Parameter & Mean & Variance & Description \\
\hline 1 & $\theta_{n}$ & 4 & 1 & Number of initial cases \\
\hline 2 & $\theta_{r}$ & $1 / 3$ & $1 / 16$ & Proportion of resistant cases \\
\hline 3 & $\theta_{N}$ & 66 & 0 & Population size (millions) \\
\hline \multicolumn{5}{|l|}{ Location } \\
\hline 4 & $\theta_{\text {out }}$ & $1 / 3$ & $1 / 256$ & Probability of going out \\
\hline 5 & $\theta_{\text {sde }}$ & $1 / 32$ & $1 / 256$ & Social distancing threshold \\
\hline 6 & $\theta_{\text {cap }}$ & $16 / 100000$ & $1 / 16$ & CCU capacity threshold (per capita) \\
\hline \multicolumn{5}{|l|}{ Infection } \\
\hline 7 & $\theta_{\text {Rin }}$ & 4 & $1 / 256$ & Effective number of contacts: home \\
\hline 8 & $\theta_{\text {Rou }}$ & 48 & $1 / 256$ & Effective number of contacts: work \\
\hline 9 & $\theta_{t r n}$ & $1 / 3$ & $1 / 256$ & Transmission strength \\
\hline 10 & $\theta_{i f f}=\exp \left(-\frac{1}{\tau_{i n f}}\right)$ & $\tau_{\text {inf }}=4$ & $1 / 16$ & Infected period (days) \\
\hline 11 & $\theta_{c o n}=\exp \left(-\frac{1}{\tau_{c o n}}\right)$ & $\tau_{\text {con }}=4$ & $1 / 16$ & Infectious period (days) \\
\hline 12 & $\theta_{i m m}=\exp \left(-\frac{1}{\tau_{i m m}}\right)$ & $\tau_{i m m}=16$ & 0 & Period of immunity (months) \\
\hline \multicolumn{5}{|c|}{ Symptoms } \\
\hline 13 & $1-\theta_{d e v}=\exp \left(-\frac{1}{\tau_{i n c}}\right)$ & $\tau_{\text {inc }}=5$ & $1 / 256$ & Incubation period (days) \\
\hline 14 & $\theta_{\text {sev }}$ & $1 / 128$ & $1 / 256$ & Probability of ARDS \\
\hline 15 & $\theta_{s y m}=\exp \left(-\frac{1}{\tau_{s y m}}\right)$ & $\tau_{\text {sym }}=8$ & $1 / 256$ & Symptomatic period (days) \\
\hline 16 & $\theta_{r d s}=\exp \left(-\frac{1}{\tau_{r d s}}\right)$ & $\tau_{r d s}=10$ & $1 / 256$ & ARDS period (days) \\
\hline 17 & $\theta_{\text {fat }}$ & $1 / 3$ & $1 / 256$ & ARDS fatality rate: CCU \\
\hline 18 & $\theta_{\text {sur }}$ & $1 / 8$ & $1 / 256$ & ARDS fatality rate: home \\
\hline \multicolumn{5}{|l|}{ Testing } \\
\hline 19 & $\theta_{t t t}$ & $1 / 10000$ & 1 & Efficacy of testing and tracking \\
\hline 20 & $\theta_{\text {sen }}$ & $1 / 10000$ & 1 & Sensitivity of testing \\
\hline 21 & $\theta_{\exp }$ & $1 / 10000$ & 1 & Sustained testing \\
\hline 22 & $\theta_{\text {bas }}$ & $8 / 10000$ & 1 & Baseline testing \\
\hline 23 & $\theta_{\text {tes }}$ & 1 & 1 & Selectivity of testing \\
\hline 24 & $\theta_{d e l}=\exp \left(-\frac{1}{\tau_{d e l}}\right)$ & $\tau_{d e l}=2$ & $1 / 4$ & Delay in reporting test results (days) \\
\hline
\end{tabular}


not true of the first wave. In other words, it would not have been possible to suppress the first wave with testing and tracking because one would have had to have identified nearly every infected, asymptomatic person in the country and this would have required about over a million tests a week.

- Although, in principle, it is mathematically possible to defer the first wave, one would require either a very small population or a very large testing capacity. Furthermore, the efficacy of testing and tracking would have to be implausibly high, i.e., around $80 \%$.

- The differences between the United Kingdom and Germany are eminently explainable under a dynamic causal model. As might have been anticipated, Germany has a greater propensity to test; however, this testing is substantially less selective for infected individuals than in the UK. Furthermore, the component due to testing and tracking during the first wave was less evident than the United Kingdom. This means that pressure is put on other parameters to explain the remarkably low fatality rates in Germany. It appears that-or it looks as ifGermany has a population whose host factors render it more resistant to infection. Furthermore, the fatality rates in critical care are substantially lower in Germany. In short, the explanation for the reduced fatalities in Germany probably lies in clinical surveillance and management, not on their testing and tracking.

In what follows, we will look at the results of simulations that license the above conclusions and unpack these conclusions quantitatively, with a special emphasis on the mechanisms and processes leading to different outcomes.

Secondary sources (Huang et al., 2020; Kissler et al., 2020; Mizumoto \& Chowell, 2020; Russell et al., 2020; Verity et al., 2020; Wang et al., 2020) and:

- https://www.statista.com/chart/21105/number-of-criticalcare-beds-per-100000-inhabitants/

- https://www.gov.uk/guidance/coronavirus-COVID-19information-for-the-public

- http://www.imperial.ac.uk/mrc-global-infectious-diseaseanalysis/COVID-19/

These prior expectations should be read as the effective rates and time constants as they manifest in a real-world setting. For example, a four-day period of contagion is shorter than the period that someone might be infectious (Wölfel et al., 2020) ${ }^{1}$, on the (prior) assumption that they will self-isolate, when they realise they could be contagious. Although the scale parameters are implemented as probabilities or rates, they are estimated as $\log$ parameters, denoted by $\vartheta=\ln \theta$.

\footnotetext{
${ }^{1}$ Shedding of COVID-19 viral RNA from sputum can outlast the end of symptoms. Seroconversion occurs after 6-12 days but is not necessarily followed by a rapid decline of viral load.
}

\section{Dynamic causal modelling}

Figure 1 provides a schematic that summarises the dynamic causal model used for subsequent inference and simulations. This model can be regarded as a factorial extension of conventional (compartmental) epidemiological models (Friston et al., 2020a). The factorial aspect means that there are several attributes in play when trying to model the causes of mortality and morbidity. Specifically, it considers the location, infection status, symptomatology, and testing status of any individual in a population. This means that for each factor, there is a certain probability of finding someone in a particular state. Movement from one state to another is parameterised in terms of transition probabilities or rate constants. For example, the probability that I will stay in a state of being infectious can be parameterised in terms of the expected number of days that I am contagious. Crucially, the transitions among states within each factor depend upon the states of other factors. These dependencies are denoted by the dashed lines. For example, the probability that I will move from a state of having no symptoms (asymptomatic) to symptoms depends upon whether I am infected or not. Note that separating the latent or hidden causes of observable data in this way means that it is possible to be infected but have no symptoms - and vice versa. This model is formally the same as previous models (Friston et al., 2020b); however, we have introduced a fifth location state called isolation. This state is entered whenever I have symptoms or am waiting to find out whether I test positive. The key mechanism-that compels me to enter isolation - is a testing and tracking (FTTI) program that alerted me to the possibility of being infected prior to developing symptoms. In this quarantined isolation, I will remain for a given period (seven days), unless I receive notice that I have tested negative following a PCR test. Please see the Methods for a formal parameterisation of these contingencies and how testing data are generated.

Although this model may look complicated; it is a straight forward compartmental model that can be written down in terms of a Master Equation, describing the discrete time updates of the joint probability distribution over the four factors. Please see (Friston et al., 2020a). Updating the joint probability (i.e., probability over all tuples of different states) allows us to model transitions among the states of one factor that depend upon other factors.

With this model in place, one can use standard variational procedures to fit any data at hand (Friston et al., 2007). Here, we used the daily reports of new (positive) cases and deaths from Johns Hopkins University ${ }^{2}$ and supplemented this with data from the UK on the total number of tests performed ${ }^{3}$. The inversion of this model takes about a minute on a personal computer, enabling one to generate posterior estimates of the parameters and accompanying trajectories of hidden states. Figure 2 shows the results of this kind of analysis for timeseries data at

\footnotetext{
${ }^{2}$ Available from https://github.com/CSSEGISandData/COVID-19.

${ }^{3}$ Available from https://github.com/tomwhite/covid-19-uk-data.
} 


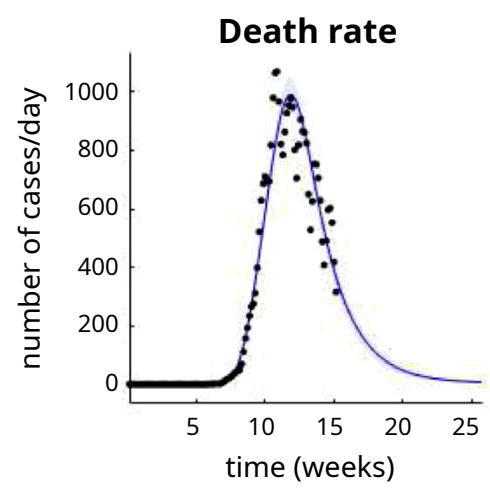

\section{Cumulative deaths}
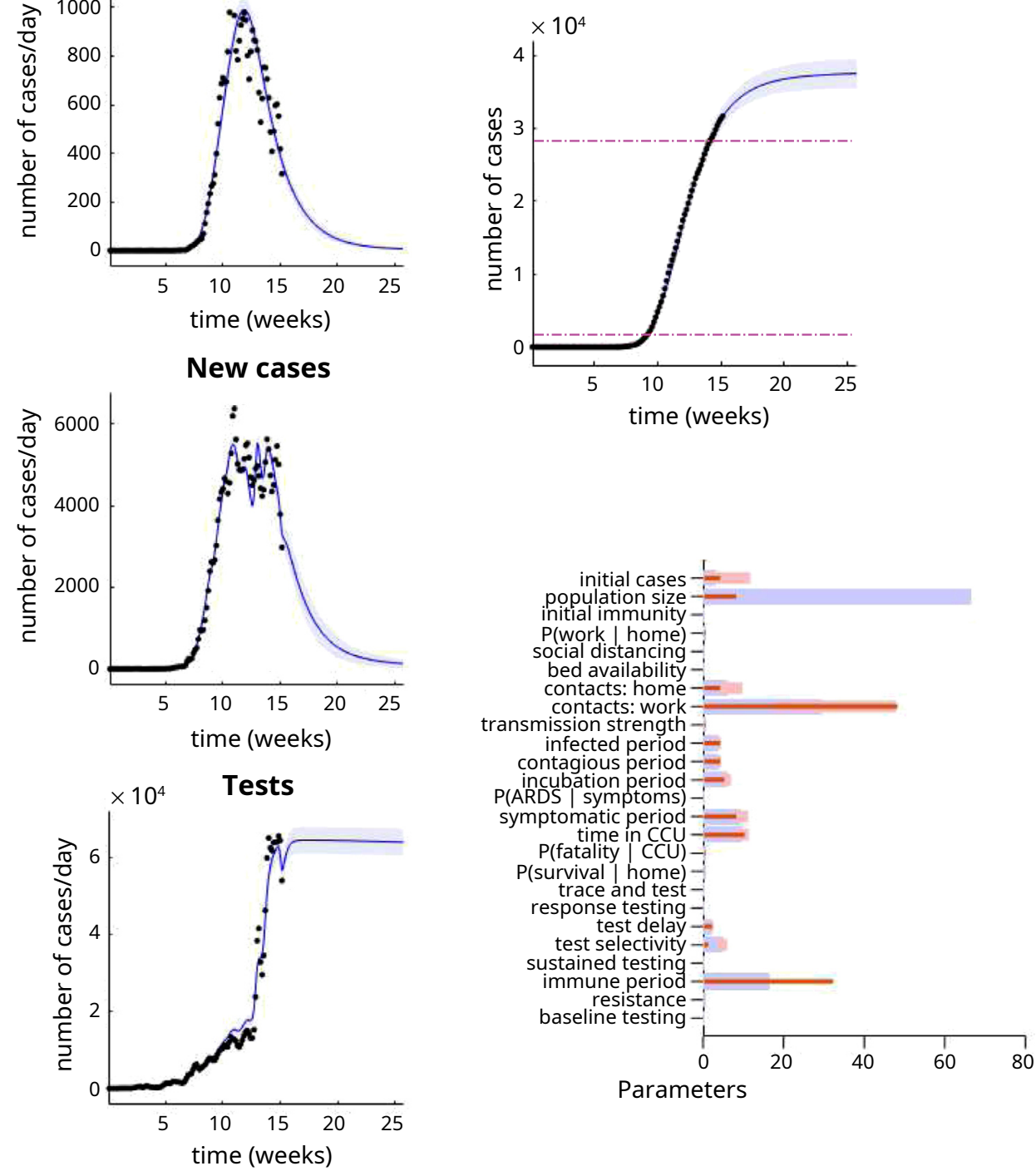

Figure 2. Posterior predictive densities. The panels on the left show the posterior predictive densities over some key outcomes. Here, daily death rates, new positive cases and tests performed. The blue lines represent the posterior expectation and the shaded areas the $90 \%$ Bayesian credible intervals. The black dots corresponds to empirical data used to fit the model and estimate posteriors over the model parameters (i.e., the transition probabilities or rate constants in Table 1). The lower right panel reports the posterior parameter densities in terms of their posterior expectation (blue bars) and 90\% credible intervals (pink bars). The red bars correspond to the prior expectations. Please see Table 1 for complete specification of the prior densities. The upper right panel shows the cumulative deaths expected under these parameters. The two dashed lines are for reference and correspond to yearly mortality rates for seasonal influenza (from $2014 / 15$ and 2018$)^{4}$.

the point of writing (10th May 2020). The left panels show the data (dots) superimposed upon a posterior predictive density. This density is a probabilistic statement about the most likely outcomes under the model. Here, it is summarised in terms of the posterior expectation or most likely outcome and $90 \%$ Bayesian credible intervals (blue lines and shaded areas, respectively). The parameters upon which these predictions are based are shown in the lower right panel. The outcomes can either be expressed in terms of daily rates or cumulative outcomes over time; for example, the cumulative deaths over a six-month period (as shown on the upper right panel). Notice

\footnotetext{
${ }^{4}$ Public Health England estimates that on average 17,000 people have died from the flu in England annually between 2014/15 and 2018/19. However, yearly deaths vary widely, from a high of 28,330 in $2014 / 15$ to a low of 1,692 in 2018/19 (dashed red lines in Figure 2). Please see Public Health England annual flu reports here: https://www.gov.uk/ government/statistics/annual-flu-reports
} 
that these posterior predictive densities cover the past and the future. In other words, they are generated from parameter estimates that do not change in time. This means that one can regard these results as a best fit to the observed data to date. Alternatively, they can be regarded as a forecast of the future. These results suggest that we are nearly halfway down the decline in daily death rates, following the peak (in early April). Crucially, because we have a generative model underneath these data, we can also generate data that has not yet been observed.

Figure 3 provides two examples of this, in terms of the effective reproduction rate $(\mathrm{R})$ and the prevalence of immunity in the left and right panels, respectively. The prevalence of immunity (a.k.a. herd immunity) is interesting because it is potentially measurable, if we had serological testing of sufficient sensitivity and specificity (Winter \& Hegde, 2020; Yong et al., 2020). When these data become available, they then can be used to improve the posterior estimates of the parameters and shrink uncertainty about the trajectory of seroprevalence (Vespignani et al., 2020) or immunity (Kissler et al., 2020).

Notice that the reproduction rate is treated as an outcome. This is an important conceptual point. The reproduction rate is not a cause of fatality-it is a measure or consequence of the underlying causes. These can be computed from the changes in the prevalence of infection and the expected duration of being contagious (please see Methods). Here, the reproduction rate starts at just under three and then falls quickly at the onset of social distancing to about 0.6 . In the future, it will gently rise as herd immunity is lost and may ultimately foreshadow a second wave (see below). The yellow line corresponds to the best available estimates of the reproduction ratio based on hierarchical Bayesian Regression, using specific known covariates (regressors) corresponding to different stages of lockdown (Flaxman et al., 2020).
These estimates ${ }^{5}$ are the kind of numbers used to currently to guide governmental policy in the UK. They can be regarded as the best estimates from state-of-the-art curve fitting. The key observation here is that they necessarily depend upon data that have already been observed. In other words, they summarise the recent past. This can be seen by comparing the yellow line with the blue line in Figure 3. The real-time estimates afforded by dynamic causal modelling (blue line) evince a more nuanced decline that precedes the sharp drop in conventional estimators (yellow line). This speaks to the potential advantage of using estimates of late states to furnish real-time or instantaneous estimates of the reproduction rate (as opposed to curve fitting or Bayesian regression estimators).

Figure 4 reproduces the results above (in the upper panels) and supplements these outcomes with the latent causes or hidden states that correspond to the factors in Figure 1. Here, we have shown the course of the pandemic over 18 months, as opposed to a six-month period. This illustrates the basic anatomy of the pandemic with an initial first wave, followed by a second wave some 36 weeks (nine months) later. The timing of this second wave depends upon the rate at which immunity is lost. In these simulations, a 16-month period of immunity was assumed.

Focusing on the initial outbreak (i.e., first wave), we can see the effects of social distancing as manifest in a very small probability of being found at work during the period of lockdown (blue line in the location panel). This coincides with a large number of people self-isolating (about $60 \%$ at its highest) during this period (purple line in the location panel).

\footnotetext{
${ }^{5}$ Available from https://mrc-ide.github.io/covid19estimates/\#/download.
}

\section{Reproduction rate - $\mathbf{R}$}

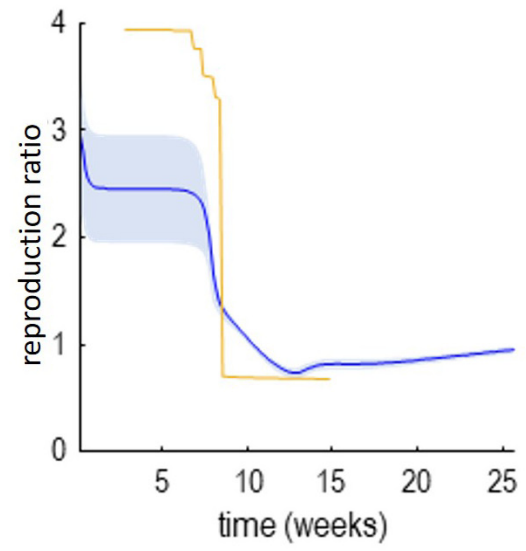

\section{Prevalence of immunity}

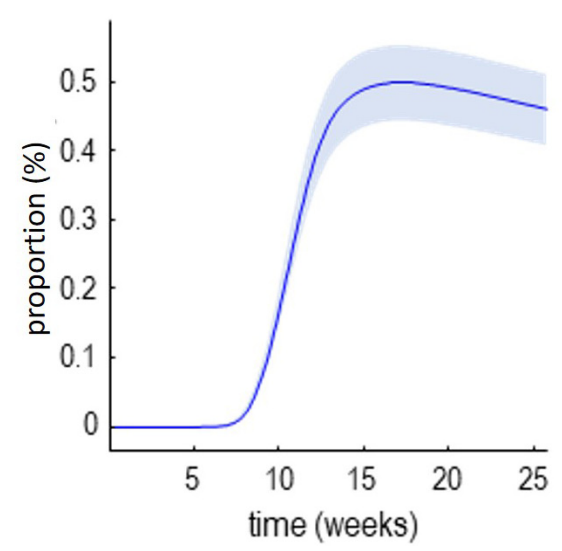

Figure 3. Reproduction rates and herd community. This figure supplements the previous figure with posterior estimates of the reproduction rate (please see Methods) and the prevalence of immunity (a.k.a. herd immunity). Again, the lines represent posterior expectations and the shaded areas $90 \%$ credible intervals. The yellow line in the right panel depicts estimates based upon a Bayesian regression model. These are the kind of estimates used to inform government policy. Please see main text for further discussion. 

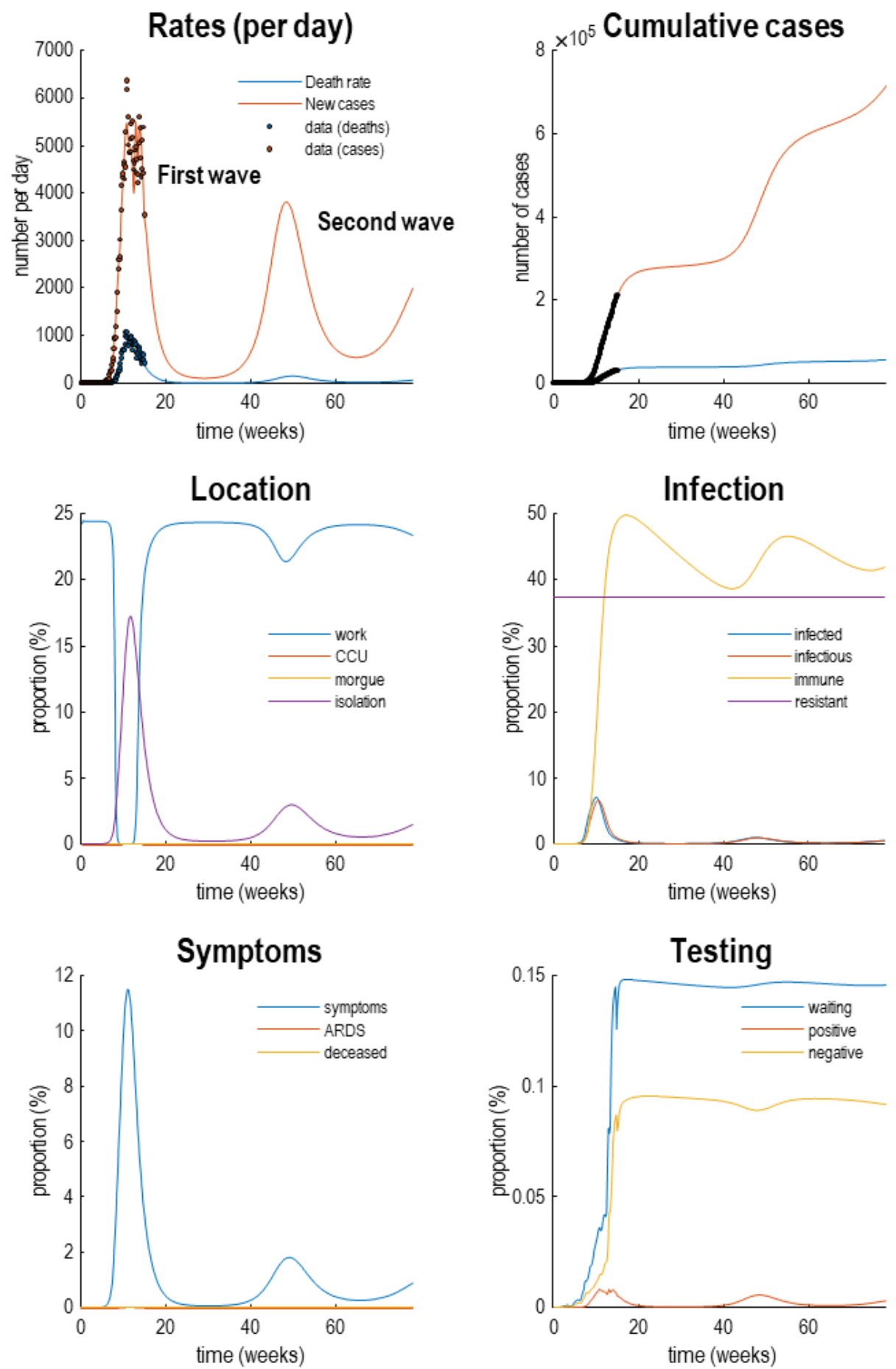

Figure 4. Latent causes. This figure shows the same data (with dots) and posterior expectations (solid lines) as in the previous figure over an 18-month period. However, here, it is supplemented with the underlying latent causes or expected states in the lower four panels. These constitute the hidden states that generate the outcomes in the upper two panels. The solid lines are colour-coded and correspond to the states of the four factors in Figure 1. For example, under the location factor, the probability of being found at work declined steeply from about $20 \%$ to 0 at the onset of the outbreak. At this time, the probability of isolating oneself rises to about $15 \%$ during the peak of the pandemic. After about five weeks, the implicit social distancing starts to relax and slowly tails off, with accompanying morbidity (in terms of symptoms) and mortality (in terms of death rate). As herd immunity (yellow line in the infection panel) declines the prevalence of infection accelerates to generate a second wave that peaks at about 48 weeks. 
Under this model, we are currently experiencing the relaxation of social distancing, with a partial return to the pre-pandemic probability of being found at work. However, the world to which we return is differs from before the lockdown. This is because a substantial number of people have acquired immunity, in virtue of being infected (whether or not they show any subsequent symptoms). The acquisition of herd immunity is depicted by the yellow line in the infection panel. Although there are no data that inform these estimates, equivalent data from Germany is starting to appear. We will return to this later. Notice that a substantial proportion of the population (about 38\%) have been estimated to be resistant. In other words, they have geographical or host factors that render them incapable of participating in the pandemic. For example, they may be isolated geographically $^{6}$ or may have genetic or developmental factors that preclude infection and viral shedding. In terms of symptoms, the most prevalent expression of the pandemic is in terms of symptoms that accompany an increase in self-isolationand pre-empt a small number of people who go on to develop a severe syndrome (e.g., acute respiratory distress) from which they may not recover. The testing panel shows the progressive increase in people waiting to be tested (blue line) that subsumes people who are subsequently positive and negative. Initially, the number of negative tests is about twice the number of positive tests, but this ratio increases as the number of infected people in the population declines. The question is: what would happen to these trajectories under different monitoring or testing policies over the next few months?

Figure 5 provides an analytic answer to this question in terms of the effects on daily death rates-as a function of time-as testing parameters are adjusted. Here, we enhanced the parameters that underwrite tracing and tracking (blue line), testing sensitivity (red line), testing delay (yellow line), testing selectivity for infected people (purple line) and, finally, the baseline probability of being testing (green line). The upper panel shows the effect on daily deaths when each of these parameters is increased by a scaling factor of one natural unit (i.e., 2.72). The key thing to observe is that the effect of changing these parameters itself changes over time. Here, we considered a period of 18 months; under the assumption that by the end of this period, there will be an effective vaccination program or other therapeutic advances.

There are two key things to note from this sensitivity analysis. First, the effect of any testing parameter on the first peak (before the vertical blue line), relative to the second peak. This second peak emerges because of a loss of immunity, modelled here with an immune period of 16 months (see Table 1). The second thing to note is that the effects are biphasic in nature. For example, increasing baseline testing initially decreases death rates with both the first and second waves, but increases death rates after the waves peak. At first glance, this may seem

\footnotetext{
See https://www.esade.edu/itemsweb/wi/research/ecpol/EsadeEcPol_ Insigth6_Exit_Strategy.pf for a discussion of isolation in terms of social networks and green zones.
}

counterintuitive; however, there is a simple explanation. This rests on the fact that any surveillance measure has the effect of delaying the spread of the virus, such that the onset of successive waves of infection is suppressed and the peak is deferred or pushed into the future. In other words, increased surveillance affords the opportunity to reduce the spread of the virus, such that successive waves of infection are delayed and dispersed-along the lines of the 'curve flattening' notion. Indeed, this was the primary motivation for social isolation to avoid excess mortality due to a saturating clinical care capacity. However, in the absence of any limitations on critical care, surveillance, in and of itself, cannot attenuate the eventual spread of the virus throughout the population-it can only delay the spread. Metaphorically, this process can be imagined as rain falling from clouds. Eventually, the downfall will reach the sea. The only thing that one can do is to moderate the flow of water and mitigate against flood damage.

Quantitatively, this key point is reflected in the overall number of lives that will be saved by enhancing one aspect of surveillance or another. The lower panels in Figure 5 shows the cumulative number of lives saved under the five different parametric enhancements. As might be expected, increasing surveillance in various ways generally decreases the cumulative deaths; however, these effects are quantitatively very small (in the tens for an effect after the first wave (left panel) and in the hundreds after the second wave (right panel). This suggests that the utility of enhanced surveillance (e.g., tracing and tracking) can only be manifest if the second wave is pushed sufficiently far into the future that it is rendered innocuous through vaccination or other therapeutic interventions.

This is illustrated in Figure 6 which simulates the trajectories that one might expect when increasingly efficacy of testing and tracking (see the Methods for how efficacy is parameterised). This figure uses the same format as Figure 4 but reproduces trajectories under increasing levels of testing and tracking. In brief, one can see is that there is hardly any effect on the first wave in terms of social distancing (location), prevalence of infection (infection), or morbidity (symptoms). However, the peak of the second wave is shifted progressively later in time, until it disappears beyond the 18-month time horizon simulated here. These simulations, as noted above, used a loss of immunity with a time constant of 16 months. This may be a somewhat pessimistic estimate of the rate at which we lose immunity; however, it clearly demonstrates the utility of testing and tracking under this (arguably worst-case) scenario. In summary, as the efficacy of testing and tracking increases, the second wave is progressively deferred, and the number of positive cases detected in the population rises. These effects are highlighted with the blue and orange arrows. So, what levels of testing and tracking would be necessary to preclude a second wave within a time horizon of 18 months?

Figure 7 answers this question by plotting the cumulative deaths and peak testing rates as a function of the efficacy of tracking and tracing. These posterior predictions were based on increasing the efficacy of testing and tracking from 0 to 1 in 

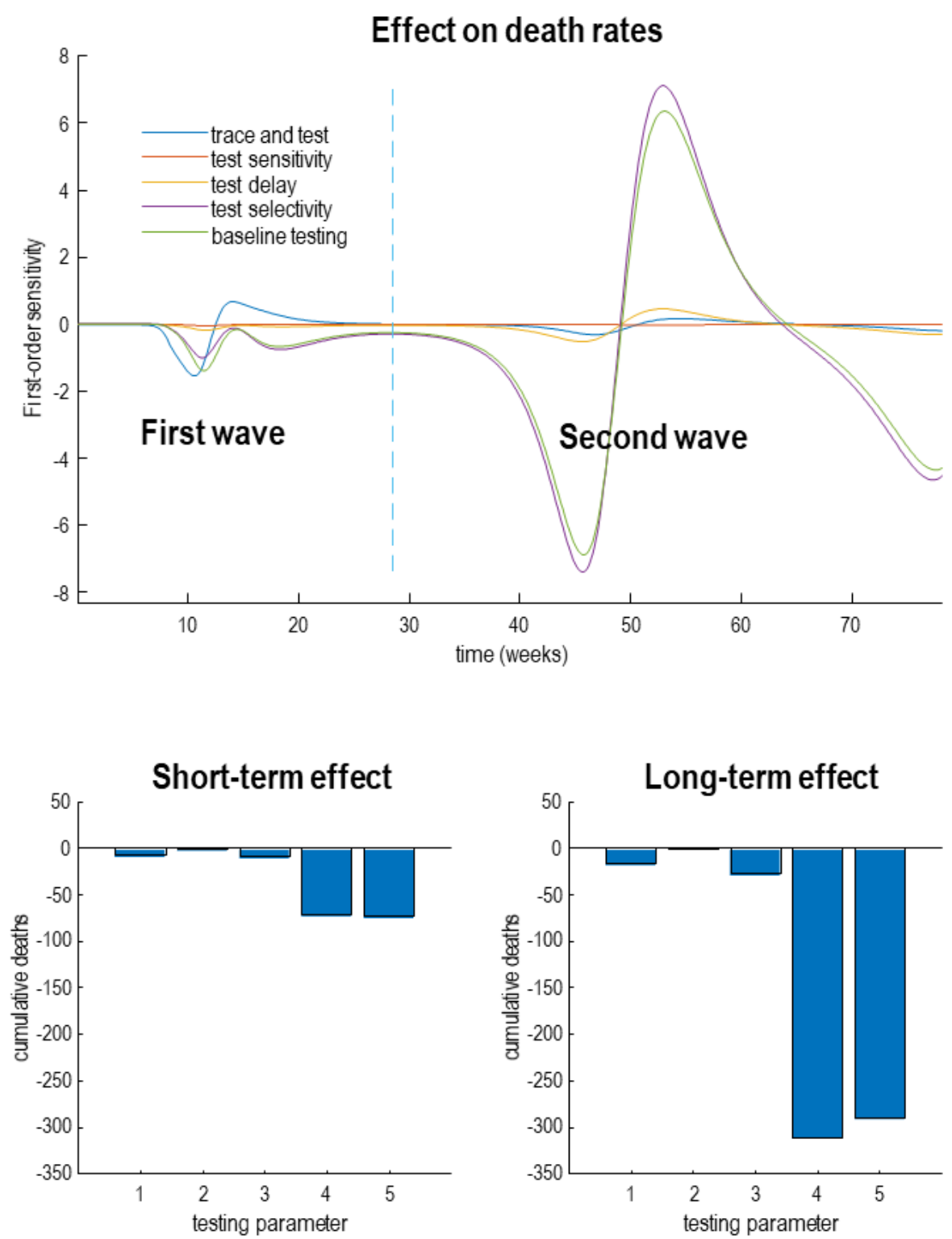

Figure 5. Sensitivity analysis. This figure illustrates the influence on death rates of various parameters that model diagnostic surveillance or testing. The upper panel shows the change in death rates with respect to the logarithm of the parameters controlling the efficacy of tracing and tracking (blue line) PCR testing in response to increasing levels of infection (red line), delay in reporting test results (yellow line), the selectivity for people who are infected (purple line) and baseline testing (green line). The lower panel shows the cumulative sensitivities or changes over time. The lower left panel sums these changes over a 20-week period prior to the onset of the outbreak, while the lower right panel accumulates the derivatives over a period of 18 months. The key thing to observe here is that the effect of changing testing or surveillance parameters is more marked during the second wave, relative to the first. Furthermore, note that the change in accumulated deaths, with respect to a unit change in log parameters is very small (in the hundreds as opposed to the thousands). This reflects the fact that the effect of these testing parameters is to shift the curve, not to attenuate its amplitude.

32 steps-depicted every four steps in the previous figure. As the efficacy of tracking and tracing increases there is a marked reduction in cumulative deaths in the order of 10,000 people. This reflects the delay in the second wave (solid line). Quantitatively, it would be sufficient to have an efficacy of about $24 \%$ to defer the second wave until 18 months. According to these estimates, this would entail peak testing rates of less than
10,000 tests per day, well within the reach of current testing capacity.

The dotted lines show the corresponding predictions for a testing and tracking strategy that was instantiated prior to the first wave. These results are interesting in the sense they speak to what would have happened had the UK government pursued 

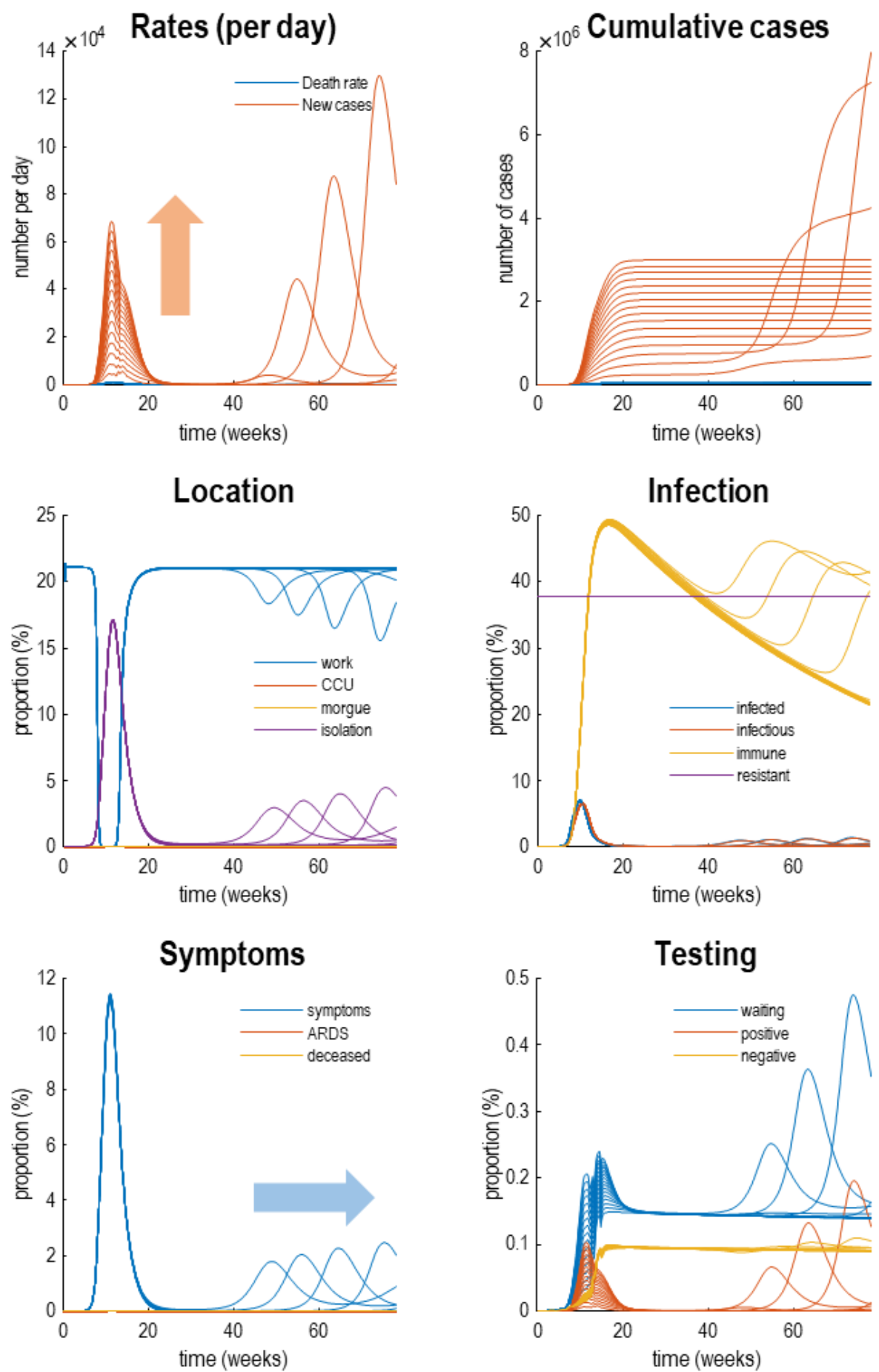

Figure 6. Testing and tracking. This figure reports the results of simulations under different protocols for testing and tracking. Specifically, we increased the probability of testing people who were infected but asymptomatic after the first wave. This increase in efficacy was from 0 to 1 in 32 steps. The results are shown using the format of Figure 4, for every fourth step. The key thing to take from this figure is that as one increases the efficacy of testing and tracking, the second wave is deferred or postponed beyond a time horizon (here, 18 months). At the same time, the total number of detected (positive) cases per day increases. These effects are summarised by the blue and orange arrows, respectively. 

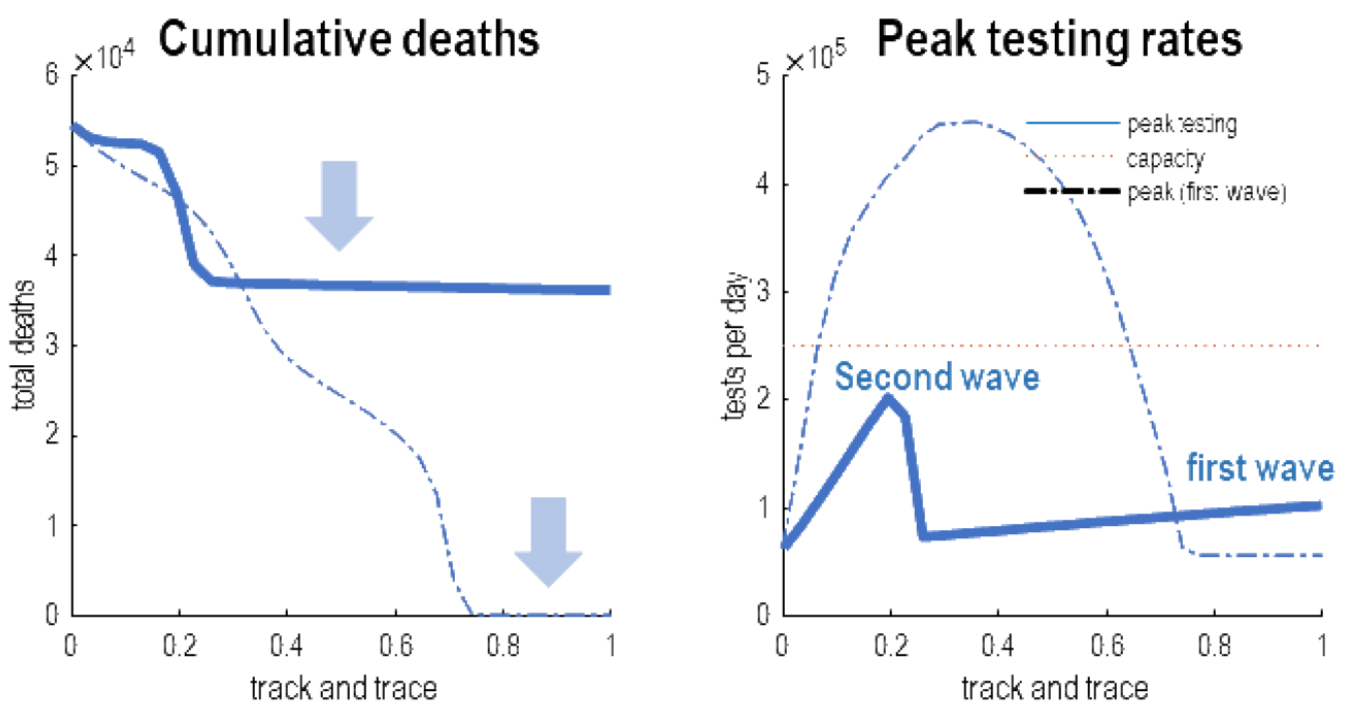

Figure 7. Suppressing waves. This figure summarises the results of the previous figure in terms of cumulative deaths after an 18-month period, as a function of the efficacy of testing and tracking. The left panel shows the total number of deaths as a function of the efficacy of a FTTI protocol that starts after the first wave (solid line) or before the first wave (broken line). The equivalent results are shown in the right panel in terms of the requisite peak testing rates over the course of the outbreak. The important thing to note is that that many lives, in principle, could be saved by eluding a second wave, provided the efficacy of FTTI exceeds about $24 \%$. This is sufficient to defer or delay the second wave until it can be rendered innocuous (e.g. through the deployment of an efficacious vaccine). The suppression of the second wave is shown by the upper blue arrow. The lower blue arrow highlights the equivalent effect on the first wave had testing and tracking been implemented at the onset of the outbreak - and maintained at efficacy levels of over $70 \%$. However, the requisite number of tests per day for intermediate level of efficacy may well have exceeded logistic capacity. This is illustrated by the red line in the right panel (here, 250,000 tests per day). In short, if one had a very small country or exceedingly well developed FTTI resources, it would have been possible to eliminate the first wave; however, for a country like the United Kingdom, this would probably not have been a practical option.

a testing and tracking strategy at the onset of the pandemic. In principle, there was a potential to defer the first wave and thereby elude any deaths due to COVID-19. This is shown by the second arrow in the left panel of Figure 7. However, things are not quite that simple. In order to eliminate the first wave, it would have been necessary to have an efficacy of testing and tracking of about $80 \%$ or more. In other words, nearly everybody who was infected but asymptomatic would have to have been identified. A more realistic efficacy of $50 \%$ would have reduced deaths in the initial phases of the outbreak; however, this would have required peak testing rates beyond the capacity of a country like the United Kingdom. This is illustrated by the dashed line in right panel that surpasses an arbitrary threshold of 250,000 tests a day. In short, although a suppression strategy based on testing and tracking is a theoretical possibility, it cannot be realised after the number of infected people exceeds testing capacity. It is interesting to speculate what this means for countries like South Korea and Singapore who have managed to elude a substantive first wave. In virtue of the fact that they have not acquired a meaningful herd immunity, they may have to maintain a high level of efficacy of FTTI, in conjunction with strict border controls and accompanying quarantine. From this perspective of the United Kingdom, the question is: do the same mechanics of surveillance apply to the second wave?

The answer to this question is no. This is because the context in which the second wave manifests is very different from the first wave. This follows because of the acquisition of herd immunity, which means that the spread of the virus-in the run-up to the second wave-is substantially attenuated. In turn, this means that the requisite efficacy of FTTI is substantially smaller. This point is illustrated in Figure 8 by evaluating the predicted outcomes at the (18-month) time horizon under four scenarios. The first scenario was a scenario based upon the posterior estimates of the testing parameters. The second scenario entailed an enhanced baseline testing. The third scenario was an enhancement of selective testing; namely, increasing the relative probability of testing infected people. Finally, we consider a FTTI strategy, in which the efficacy was increased from near zero to $25 \%$. The upper panels show the posterior predictions as a function of time (left panel) and as a phase-space summary of the same trajectories (right). This way of detecting trajectories plots one outcome against another: i.e., plotting the daily rates of new cases against daily deaths.

The resulting trajectories illustrate effects of various interventions. In brief, as we increase the rate or selectivity of testing, we shift the trajectories upwards. In other words, we increase the number of detected cases but with little effect on daily deaths. In contrast, the FTTI strategy suppresses the second wave and reduces daily deaths. The lower panel quantifies the endemic endpoint at the time horizon of 18 months. This is not an endemic equilibrium but stands in for the state of affairs at the point of a presumed vaccination or therapeutic intervention. 


\section{Predicted deaths}

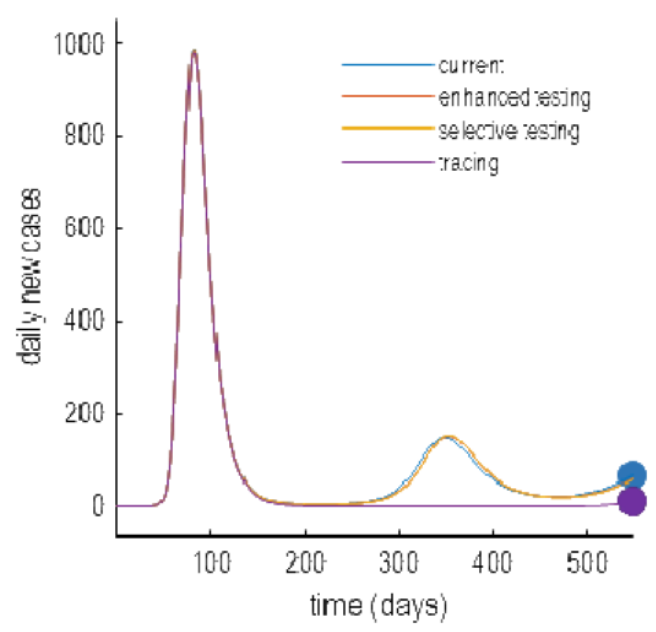

Trajectories

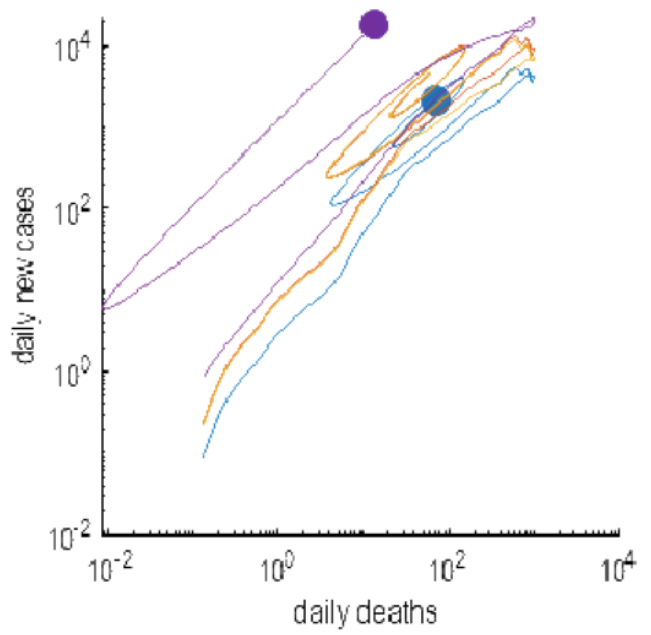

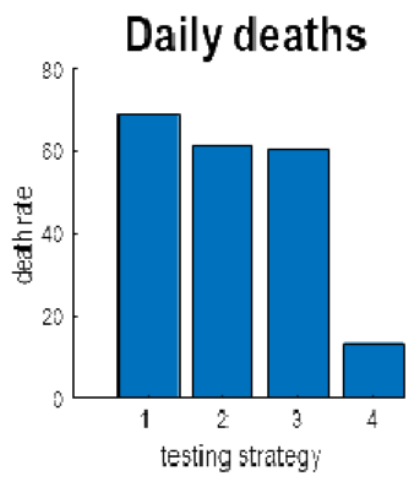
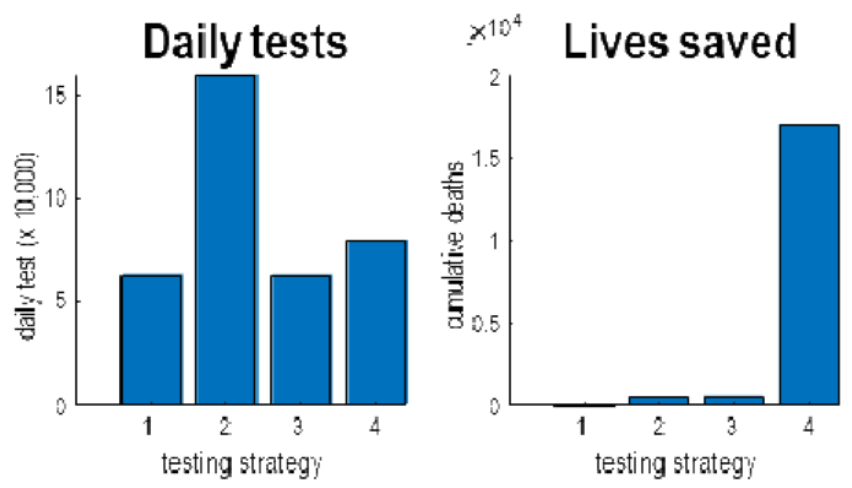

Figure 8. Different testing strategies. This figure reports simulations of what might happen under different testing or surveillance regimes. The upper panels show the simulated daily deaths predicted under four strategies. These include the current strategy based upon the posterior expectations of testing parameters. The red lines show the response to an enhanced baseline testing. The yellow lines simulate the outcomes under an increase in selective testing, while the green lines illustrate the impact of testing and tracking within efficacy of 25\%. For the enhanced testing, the posterior expectations of the log parameters were increased by a value of one (i.e., the scale parameters were multiplied by 2.72). The FTTI parameter was increased to a value of one quarter. The upper left panel plots the predicted deaths as a function of time for an 18-month period. The upper right panel shows the same data but plotted as a trajectory in a phase space, spanned by daily deaths and reports of new cases. This illustrates the fact that, mathematically, the trajectories into the future correspond to flows towards an attracting orbit or set. In this instance, the systems have point attractors. However, here, we have assumed that a vaccine is available at 18 months, at which point the trajectories terminate in the filled circles. The lower three panels characterise this endpoint in terms of daily deaths, daily tests and total number of lives saved since the onset of the outbreak. It can be seen that, as might be anticipated, the successive enhancements of testing reduce daily deaths. The most expensive strategy, in terms of daily tests, is the enhanced testing strategy requiring 160,000 tests again. The remaining strategies require a more modest 70,000 tests per day. The most efficient and lifesaving strategy is the implementation of testing and tracking that could, under this model, said more than 15,000 lives. The blue dots in the upper panels denote the endpoints that, here, stand in for the endemic equilibria under the four strategies. Note that the testing and tracking strategy is the only its approach that materially decreases daily deaths, both over time and at the endpoint.

One can see that the various testing strategies progressively reduce the daily death rates at this endpoint. For example, with an FTTI efficacy of $25 \%$, the daily deaths due to COVID-19 are about 10 per day. This is roughly the number of people who are killed in road traffic accidents ${ }^{7}$. The number of tests

https://www.gov.uk/government/collections/road-accidents-and-safetystatistics. at this time, are reasonably manageable (about 50,000 per day for the FTTI strategy). Crucially, the number of lives saved is reduced considerably under, and only under, FTTI. In this example, the elimination of the second wave would save about 16,000 lives. Notice that simply elevating the sensitivity or selectivity of testing has little effect on the mortality rates. Only the FTTI strategy enables the early identification of infected individuals, their subsequent isolation and ensuing deferment of a putative second wave. 
An important (if obvious) observation, implicit in this treatment, is that testing can be deployed in different ways with distinct agendas. Crucially, the only kind of testing that matters for saving lives is identifying those individuals who are infected before they can spread the virus. This is the raison d'être for FTTI, as opposed to simply increasing test rates. Increasing the baseline, sensitivity or selectivity of testing provides more precise data for epidemiological modelling and subsequent policy decisions; however, in and of itself, it will not have any material effect on the progression of the pandemic. Similarly, testing people who are symptomatic is too late from the point of view of isolating individuals who may become contagious-even if it allows people to return to work early. Clearly, all three agendas are important; however, it may be useful to consider (and model) testing in terms of its distinct aims; namely, to defer a second wave, to enhance epidemiological surveillance and to ease pressure on the economy and clinical care.

\section{A comparative analysis}

The conclusions from the above modelling are clear. There is an imperative to instantiate (or possibly re-instantiate) FTTI at modest levels of efficacy in the next few months-to preclude a second wave by delaying it. Furthermore, trying to maintain an early FTTI strategy at effective levels would have beenlogistically difficult. This begs the question: how has Germany managed to suppress its mortality rates, if it contended with the same kind of outbreak confronting the United Kingdom? To answer this question-at the fairly crude level-we repeated the dynamic causal modelling using daily new cases and deaths from Germany. The trajectories of these outcomes and their latent causes are shown in the upper and lower panels of Figure 9, respectively. Because there was no available data on the total number of tests, we assumed a constant baseline testing.

The ensuing differences in the outcomes and latent states speak to what we already know. For example, despite having about the same number of people testing positive during the first peak, the mortality rates in Germany are about a quarter of those witnessed in the UK. The inferred surveillance and testing suggest that Germany started with a baseline testing rate, such that at any one time $0.1 \%$ of the population was waiting for their test results. The UK, conversely, accrued its testing capacity during the first wave and, according to these estimates, now exceeds the German testing rates. Despite increased testing in Germany, the number of people selfisolating was about half that in the UK (as estimated under this model), with less than $10 \%$ of the German population quarantining themselves at the peak of the pandemic. Furthermore, Germany's social distancing was less stringent and shorter, as reflected by the blue lines in the location panel of Figure 9. The infection panel is telling; in the sense that about $38 \%$ of the UK population are estimated to be resistant. However, this rises to about $58 \%$ of the German population. This is a marked difference suggesting either geographical or host factors may play an important role in the differential fatality rates. Indeed, when one examines the underlying posterior parameter estimates for the trajectories depicted in Figure 9, it becomes clearer how Germany and the UK differ.
Figure 10 shows the parameters with the greatest difference between Germany and the UK, in terms of the country specific estimates (upper panels) and the differences (lower panels). The parameters are shown in terms of $\log$ parameters (left panels) and the corresponding scale parameters (right panels). The scale parameters are nonnegative rate constants and probabilities, while the log parameters are simply the log transformed scale parameters. For clarity, only the 12 parameters with the greatest posterior difference are shown. They have been ranked such that the parameters on the left show the greatest difference (the parameters are labelled by the subscripts in Table 1). The key thing to take from this comparison is that there are marked differences between Germany and the UK, both in the testing parameters and the parameters pertaining to susceptibility and clinical surveillance. Indeed, the most marked difference is a fivefold increase in the sensitivity of German testing to the prevalence of infection. This testing is nearly 5 times less selective for infected people than in the UK. This is consistent with what we know from the German approach relative to the U.K.'s approach. The third largest difference is the number of people infected $(n)$ at the beginning of the timeseries. The inference here is that Germany started with about three times as many infected people as the United Kingdom. By virtue of the fact that Germany tested more sensitively but non-selectively from the onset of the outbreak, the sustained testing component (exp) is much smaller. Note also that the FTTI parameter ( $t t t)$ is also smaller. In other words, there is no evidence that testing and tracking in Germany contributed to their surveillance program. The key parameters to note here are the substantial (about 50\%) increase in the proportion of the German population that were resistant to infection and a (about 20\%) decrease in the probability of fatality in critical care. This is despite Germany having a larger population (83 million as opposed to 66 million in the United Kingdom). Finally, the probability of developing severe symptoms when infected is slightly lower than in the UK.

The evidence here contradicts the hypothesis that Germany's relatively low fatalities are caused by a more vigorous testing programme. At one level, this hypothesis is naive because testing cannot cause morbidity. It can only detect the consequences of morbidity. As noted above, the only way that testing can affect fatalities is by delaying the spread of the virusand this is most effective when infected but asymptomatic individuals are identified through testing and tracking. A more plausible interpretation of these parameter estimates, and ensuing predictions is as follows:

The proportion of people testing positive who subsequently died in Germany is lower not because people who are infected are less likely to die but simply because Germany has tested more people. The reduced mortality rates may reflect the differential prevalence of infection in cohorts of the population that are more resistant. The relative reduction in the probability of developing severe symptoms and subsequent fatality may well reflect the clinical surveillance and management of symptomatic people. For example, anecdotal reports from respiratory physicians in Germany suggest a more 

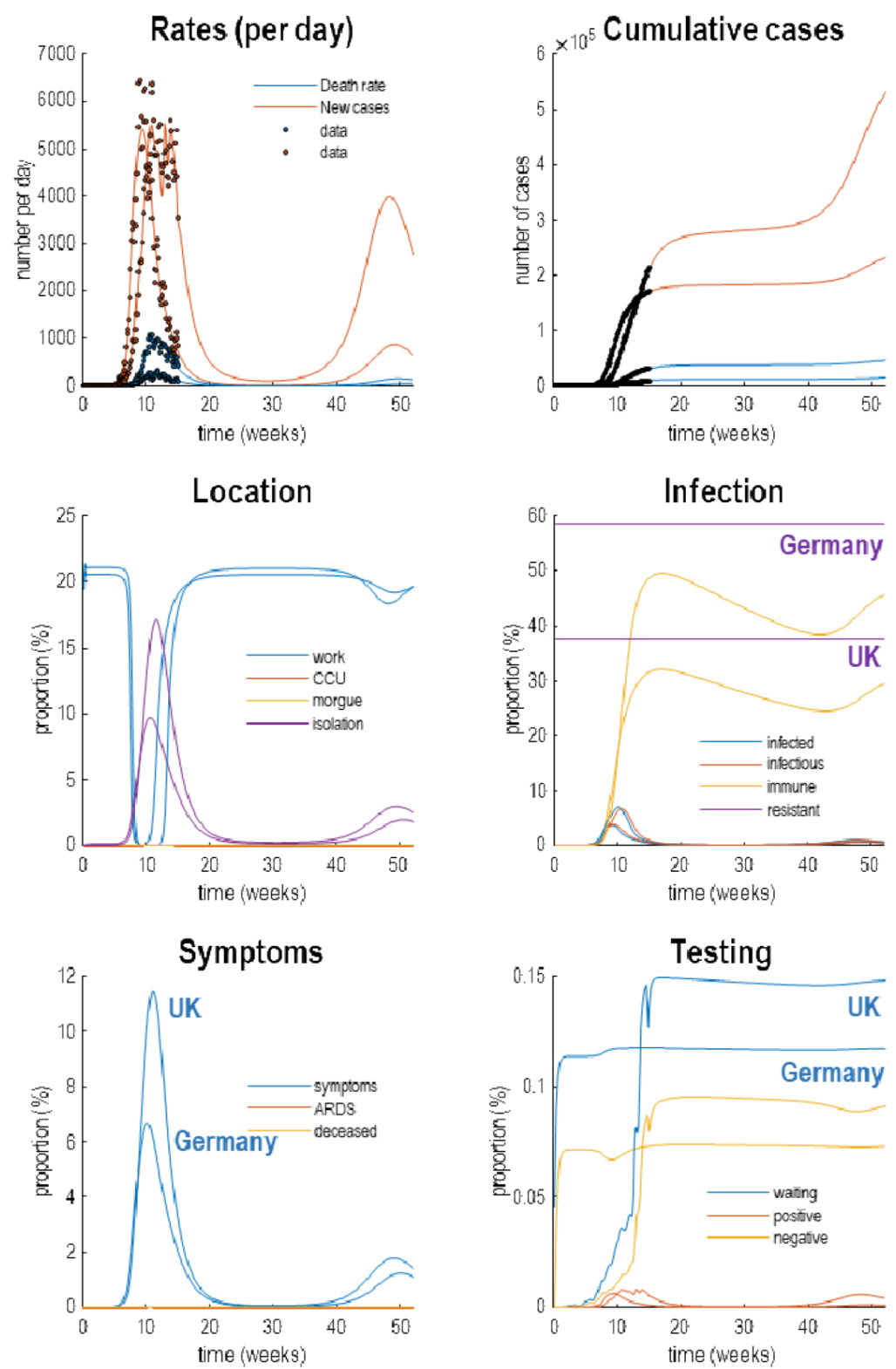

Figure 9. Germany and the UK compared. This figure shows the latent causes (lower panels) of observed and predicted outcomes (upper panels) for Germany and the UK. The coloured pairs of lines in each panel use the same format as Figure 4 and refer to the two countries in question. The generative model provides a good account of the empirical data for both countries (black dots in the upper panels), with formally similar fluctuations in latent epidemiological states. However, there are some key quantitative differences. For example, the degree of self-isolation and social distancing is attenuated by roughly one half in Germany. This pertains both to the percentage of people self-isolating and the duration of social distancing at a societal level. This is also reflected in the lower prevalence of symptomatic individuals at the first (and second) peaks of infection. These differences are, in large part, due to the number of people who are susceptible to infection, as reflected in the proportion of people who are resistant (about 38\% for the UK and 58\% for Germany). The parameters that underwrite these trajectories are shown in the next figure.

prospective clinical management, with lower thresholds for admission to critical care. In contrast, much of the disease burden in the UK appears to have been managed in an elderly and vulnerable population in care homes. This cohort are unlikely to survive the rigours of intubation in an intensive care unit and their clinical management is necessarily more palliative. In short, Germany may have had to deal with a different kind of problem than that confronting the UK. In short, although German testing and clinical surveillance was more in evidence, only the clinical surveillance mattered in terms of mortality.

Clearly, this is purely speculation; however, in principle, it should be possible to evaluate the evidence for these speculative 
hypotheses when more detailed data becomes available. This speaks to one application of dynamic causal modelling to compare different models in terms of their evidence (Penny et al., 2004); for example, comparing a model of outcomes in Germany and the UK with, and without, country-specific differences in the parameters.

\section{Conclusion}

The key conclusions from this kind of modelling are twofold. First, the hypothesis that a flareup or rebound of infections will ensue if we relax social distancing prematurely are not supported by the evidence at hand. A popular conception of this immediate (second wave) is akin to lowering the "flood gates' too soon and being overwhelmed with a deluge of infections. However, this picture may be a false impression. There can be no flood of infections because a sufficient proportion of the population have already been exposed to the virus. These people preclude a rapid spread of the virus through the population by acting as a retardant or buffer that suppresses the effective reproduction ratio. In other words, the first wave cannot flareup because it has largely exhausted the necessary substrate of susceptible individuals it needs to disseminate itself. This speaks to the second key conclusion.

Over the forthcoming months any 'flood' will be a 'trickle'. In this limited window of opportunity, FTTI protocols
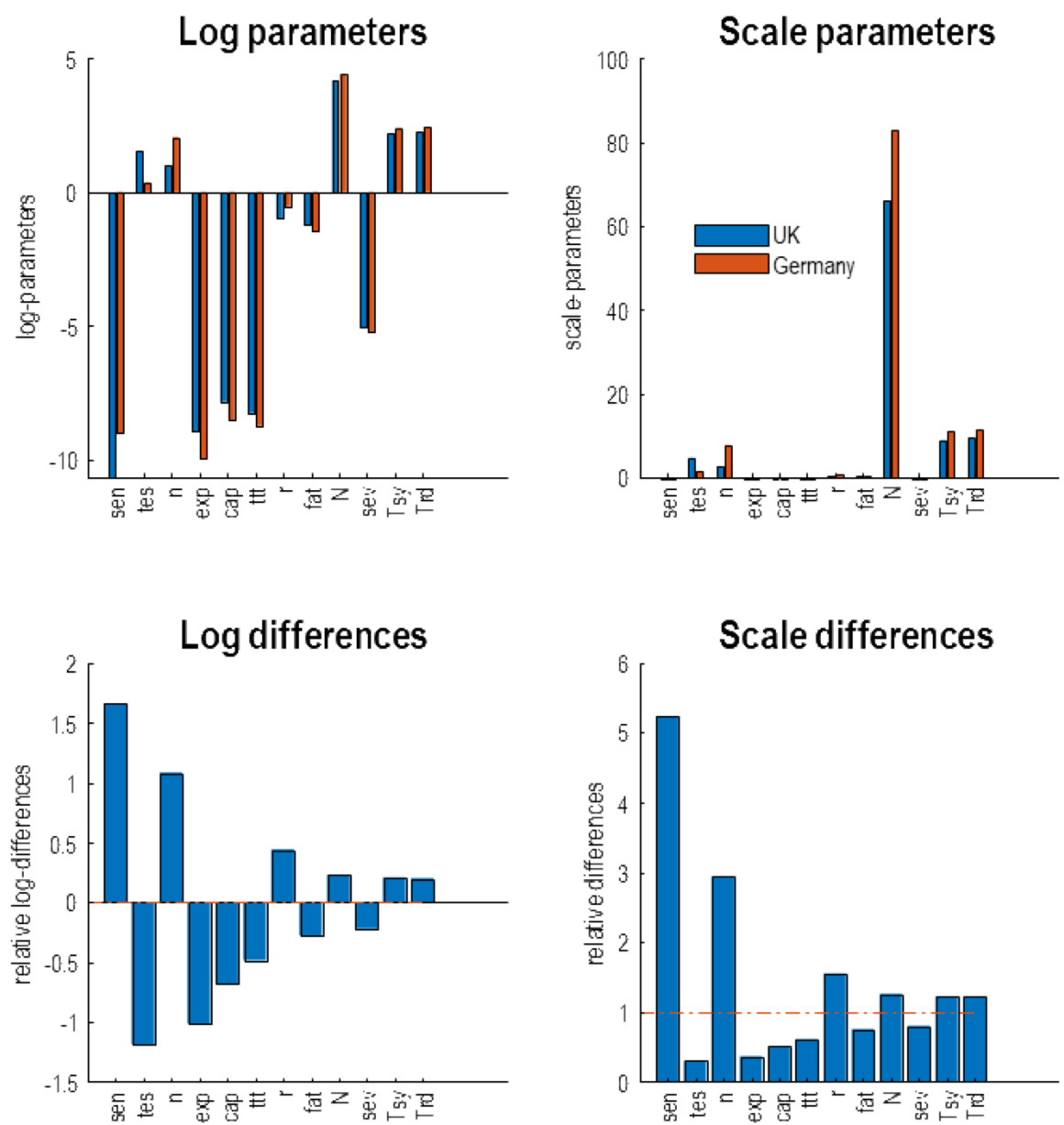

Figure 10. A parametric comparison. This figure shows the posterior expectations of the model parameters form Germany and the UK. The upper panels show the estimates for each country separately, while the lower panels show the differences. The left panels show the parameters in terms of their logarithmic form, while the right panels show the exponentiated (scale) parameters. In the upper panels, the UK parameters are in blue and the German parameters are in orange. The labels on the X axis correspond to the parameter subscripts in Table 1. The key thing to take from this figure is that the most marked (quantitative) differences between the two countries lies in the parameters pertaining to testing; namely, the sensitivity to the prevalence of infection, the selectivity of testing in terms of whether people are infected or not and the sustained testing after the first peak. Having said this, testing and tracking is actually less in Germany-as estimated by the model-than in the UK, while Germany looks (i.e., appears) as if it has a more sensitive testing or surveillance program that is less selective for people who are infected. These testing parameter differences explain why the number of positive cases reported is about the same for United Kingdom and Germany, while the latent number of people who are infected and subsequently die is much less (by a factor of roughly 4). The actual cause, according to this model, of this differential mortality lies in the clinical and management parameters. These include an increased number of resistant members of the population and a reduced fatality rate, when severely ill. Furthermore, there is a decrease probability of developing severe symptoms when infected. 
become viable, in the sense that detecting asymptomatic and infected individuals with a reasonable (e.g., 25\%) efficacy would be sufficient to delay the re-emergence of the virus-a re-emergence that rests on, and only on, a slow loss of immunity. In short, FTTI will work after the first wave, even if it was logistically viable before the first wave. If, collectively, we lose immunity over a period of many months or years, then a second wave can be eluded, via FTTI, saving thousands of lives. This is under the proviso that a second wave can be pushed sufficiently far into the future, where it is rendered innocuous by vaccination or other interventions.

Clearly, this narrative depends on the acquisition of (herd) immunity following the first wave and its subsequent loss due to population fluxes, geo-social and serological factors. The big question at the moment is whether the first wave has induced a sufficient level of herd immunity to open the window of opportunity for testing and tracking. Although there is no current data for the UK, early studies in Germany speak directly to this issue. Figure 11 reproduces the hidden states in Figure 9, with a focus on infection status. The prevalence of immunity is shown as a yellow line. At the peak of the first wave (shortly after 60 days) the inferred level of immunity is $15 \%$. This is the level of immunity estimated empirically in provisional reports of a serological study of people living in a region near Bonn (Streeck et al., 2020) ${ }^{8}$. Results of this sort are encouraging and endorse the inferences afforded by dynamic causal modelling. Perhaps more importantly, over the next few weeks more serological studies will become available and we will know with much greater certainty whether the above narrative is licensed by empirical data. If not, these data can be assimilated into the model to update our (Bayesian) beliefs about what has happened, what will happen and what could happen.

This DCM does not include the influence of repeated seeding. For example, the UK has detected introductions of SARS-CoV-2 cases, which is much higher than the assumed number of initial cases in this study (Firth et al., 2020). However, for simplicity, we have assumed that the community transmission had, quantitatively, a much greater contribution than reseeding. Having said this, it would be interesting to compare models with and without reseeding to test this assumption formally.

In this work, the model assumptions on contact patterns are simplified for e.g. age-stratified contact patterns are not considered (du Plessis et al., 2020). However, the current extensions of the model with eight stratification and contact matrices have been developed and are available in our opensource code base (DEM_COVID_S.m). Recent work on age stratified models using ONS data with age demographics now underwrites the dashboard ${ }^{9}$ based upon the current DCM

\footnotetext{
${ }^{8}$ Preliminary results from a town of about 12,500 in Heinsberg-a region in Germany that had been hit hard by COVID-19—suggest a seropositive prevalence of $14 \%$ of 1007 people assessed between March 30 and April 62020.
}

(DEM_COVID_UK.m). However, in the current model, we have simply lumped together all age groups-and have focused on heterogeneity in contact rates by conditioning them on different locations in the location factor.

Here we also note that the estimation of the true number of infected people from noisy timeseries data is a real challenge and different approaches are proposed, with very different outcomes (number of total cases from 2 times to 15 times the number of diagnosed cases), see (Bohning et al., 2020; Phipps et al., 2020). It is indeed the potential to estimate the true number of infected people (and other key latent variables) that led us to adopt and advocate the generative modelling approach of DCM, coupled with variational Bayesian inference that allows us to quantify and account for uncertainty in the case and death data. A validation of these kinds of estimates, with those provided by Edge Health, was recently reported in the Guardian $^{10}$.

\section{Methods}

\section{Modelling self-isolation}

Equipped with an extra location (isolation) state enables one to distinguish between simply staying at home or being out and about (i.e., at work). These states are rough approximations to the different kinds of environment we find ourselves in and are used to differentiate the number of contacts that could potentially transmit the virus from one person to another. When considering the parameterisation of population dynamics, in terms of being in a particular state, one has to parameterise the time spent in that state, in relation to the probability of leaving or entering it. In this instance, the probability of entering self-isolation is unity when you develop symptoms or submit to PCR testing. You then remain in that state for seven days, unless you receive notification that you have tested negative. While in this state, you can neither infect nor be infected by anybody else. Mathematically, this can be parameterised as follows, where $\tau_{i s o}$ is seven days (conditional on not being in critical care or the morgue)

$P\left(\right.$ isolation $_{t+1}$ isolation $\left._{t}\right)=\left\{\begin{array}{cc}1 & \text { if sympotomatic, positive or waiting } \\ 0 & \text { if negative and asymptomatic } \\ \exp \left(-1 / \tau_{\text {iso }}\right) & \text { otherwise }\end{array}\right.$

Clearly, the transition from one location state to another now depends upon the testing factor. If you are waiting for a test, you move into isolation and if you are negative you leave. It is this conditional dependency between the factors that mediates the efficacy of FTTI.

\section{Modelling FTTI}

The parameterisation of FTTI and other testing state transitions is a bit more delicate. This is because there are several reasons you might be tested that depend on several factors.

\footnotetext{
${ }^{9}$ https://www.fil.ion.ucl.ac.uk/spm/covid-19/dashboard/local/

${ }^{10} \mathrm{https}$ ///www.theguardian.com/world/ng-interactive/2021/jan/10/one-infive-have-had-coronavirus-in-england-new-modelling-says
} 


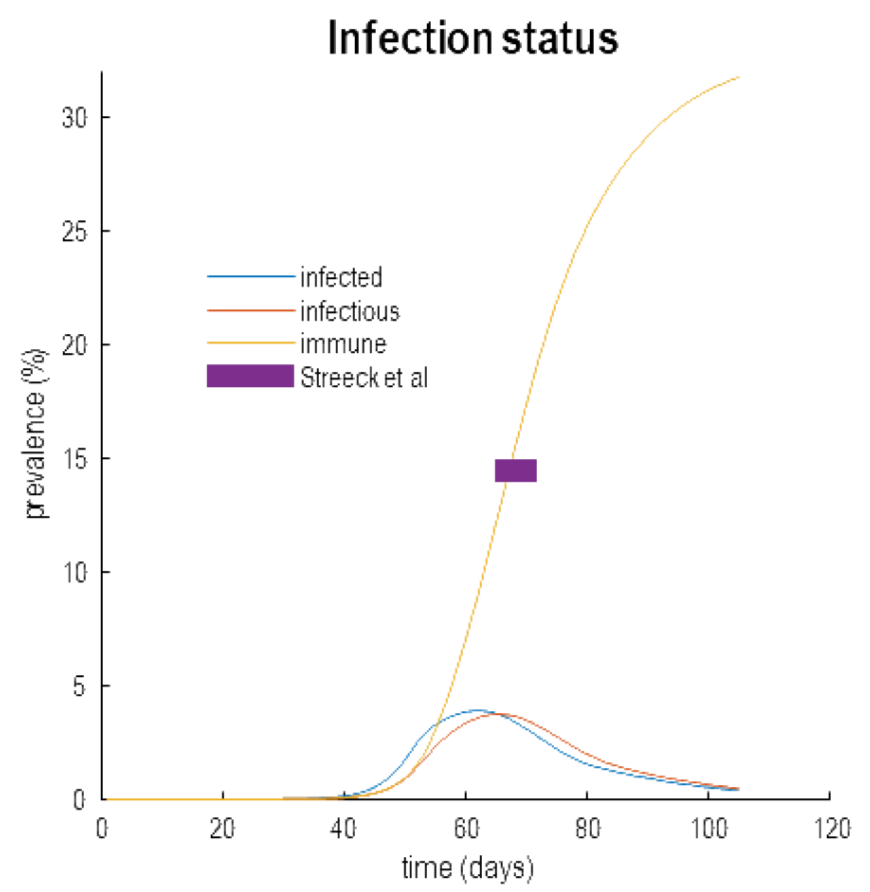

Figure 11. Herd immunity. This figure reproduces the results of Figure 9 but with a focus on infectious status. Here, the estimated prevalence of infected people, contagious (infectious) people and immune people are shown as a function of time since the onset of the outbreak (25th of January 2020). The purple bar indicates the level of estimated seroprevalence in a region in Germany as reported in streak et al. This matches the predictions of the model; namely, a 15\% herd immunity at the peak of the infection. According to this analysis, this level of herd immunity is contextualised by the proportion (about 42\%) of the population that are susceptible to infection and subsequent morbidity.

In this model, we parameterise testing by first establishing a time-dependent probability of being tested on any given day. If we now condition the probability of being tested on whether or not you are infected, we need to parameterise the relative probability of being tested when an infected, in relation to not being infected. This means that the total probability of being tested is a weighted average of two probabilities, parameterised as follows (Please see Table 1 for a description of the parameters):

$$
\begin{aligned}
P(\text { tested }) & =P(\text { tested } \mid \text { infected }) P(\text { infected })+P(\text { tested } \mid \text { not infected }) P(\text { not infected }) \\
& \Rightarrow \\
P_{\text {tes }} & =P(\text { tested } \mid \text { infected })=\theta_{\text {tes }} P_{\text {sen }} \\
P_{\text {sen }} & =P(\text { tested } \mid \text { not infected })=\frac{P(\text { tested })}{\theta_{\text {tes }} P(\text { infected })+1-P(\text { infected })}
\end{aligned}
$$

where the probability of being tested has three components:

$$
P(\text { tested })=\theta_{\text {bas }}+\theta_{\text {sen }} P(\text { infected })+\theta_{\text {exp }} P(\text { immune })
$$

The first is a baseline probability. For the UK analyses, this parameter was proportional to the total number of tests up until the present time, and the maximum number of tests in the future. The second parameterises a sensitivity to the prevalence of infection in the community and increases with infection rates. The final term is a sustained response following onset of the first wave. Here, we use the level of immunity as a proxy for a gently declining function of the cumulative number of affected people. Each of these terms has a parameter enabling one to fit a time-dependent probability of being tested. Finally, we have to consider targeted testing of individuals who have been identified as having been in contact with an infected individual. This affords an enhanced probability of testing if, and only if, you are infected and asymptomatic. By adding this probability to the probability of being tested when infected, we supplement general screening with a FTTI parameter as follows:

$P($ tested $\mid$ infected, asymptomatic $)=P_{\text {tes }}+\theta_{t t t}\left(1-P_{\text {tes }}\right)$

The ensuing parameter is simply the efficacy or extra probability that I will be tested if I am infected and asymptomatic. If it were possible to trace and test everybody who has been exposed and contracted the virus prior to developing symptoms, this efficacy will be one. In the absence of any targeted testing efficacy will be zero. A priori, the efficacy was set to very low levels of one in 10,000 people, per day.

\section{Effective reproduction rate}

The effectively production rate is a fundamental epidemiological constant that provides a useful statistic that reflects the exponential growth of the prevalence of infection. There are 
several ways in which it can be formulated and estimated. For our purposes, we can generate an instantaneous reproduction rate directly from the time varying prevalence of infection as follows:

$$
\begin{aligned}
& R_{t}=\exp \left(K_{t} \cdot \tau_{\text {con }}\right) \\
& K_{t}=\ln \frac{P\left(\text { infected }_{t+1}\right)}{P\left(\text { infected }_{t}\right)}=\frac{\ln (2)}{T_{d}}
\end{aligned}
$$

These expressions show that the reproduction rate reflects the growth of the (logarithm of) proportion of people infectedand the period of being infectious. This number is formally related to the doubling time $T_{d}$. Note that the reproduction rate is not an estimate in this scheme: it is an outcome that is generated by the latent causes or hidden states inferred by inverting (i.e., fitting) the model to empirical timeseries.

\section{Software note}

The annotated (MATLAB/Octave) code is available as part of the free and open source academic software SPM (https:// www.fil.ion.ucl.ac.uk/spm/), released under the terms of the GNU General Public License version 2 or later. The routines are called by a demonstration script that can be invoked by typing DEM_COVID_T at the MATLAB prompt. For this technical report, we used MATLAB R2019b and SPM12 r7850 (archived at https://doi.org/10.6084/m9.figshare.12174006.v4 (Friston et al., 2020c)).

We recommend anyone interested in applying this model should use the latest version of the software available. Details about future developments of the software will be available from https://www.fil.ion.ucl.ac.uk/spm/covid-19/.

\section{Software availability}

Software is available from: https://www.fil.ion.ucl.ac.uk/spm/ covid-19/

Archived source code at time of publication: https://doi.org/ 10.6084/m9.figshare.12174006.v4 (Friston et al., 2020c)

License: GLP 2.0+

\section{Data availability}

\section{Source data}

The data used in this technical report are available for academic research purposes from the COVID-19 Data Repository by the Center for Systems Science and Engineering (CSSE) at Johns Hopkins University, hosted on GitHub at https:/github.com/ CSSEGISandData/COVID-19 and from the Coronavirus (COVID-19) UK Historical Data repository by Tom White hosted on GitHub at https://github.com/tomwhite/covid-19-ukdata and also from figshare.

\section{Underlying data}

Figshare: Dynamic Causal Modelling of COVID-19. https://doi. org/10.6084/m9.figshare.12174006.v4 (Friston et al., 2020c)

This project contains the following underlying data:

$$
\text { - covid-19-tests-uk.csv (UK COVID-19 historical data) }
$$

Data are available alongside the source code under a GLP 2.0+ license.
Aleta A, Martin-Corral D, Pastore Y, et al.: Modeling the impact of social distancing, testing, contact tracing and household quarantine on secondwave scenarios of the Covid-19 epidemic. medRxiv. cidid. 2020. Publisher Full Text

Bohning D, Rocchetti I, Maruotti A, et al.: Estimating the undetected infections in the Covid-19 outbreak by harnessing capture-recapture methods. Int J Infect Dis. 2020; 97: 197-201.

PubMed Abstract | Publisher Full Text | Free Full Tex

du Plessis L, McCrone JT, Zarebski AE, et al.: Establishment \& lineage dynamics of the SARS-CoV-2 epidemic in the UK. medRxiv. 2020. Publisher Full Text

Ferretti L, Wymant C, Kendall M, et al.: Quantifying SARS-CoV-2 Transmission Suggests Epidemic Control With Digital Contact Tracing. Science. 2020; 368(6491): eabb6936.

PubMed Abstract | Publisher Full Text | Free Full Text

Firth JA, Hellewell J, Klepac P, et al.: Using a real-world network to model localized COVID-19 control strategies. Nat Med. 2020; 26(10): 1616-1622. PubMed Abstract | Publisher Full Text

Flaxman S, Mishra S, Gandy A, et al.: Report 13: Estimating the number of infections and the impact of non-pharmaceutical interventions on COVID-19 in 11 European countries. 2020.

Publisher Full Text

Friston K, Mattout J, Trujillo-Barreto N, et al.: Variational Free Energy and the Laplace Approximation. NeuroImage. 2007; 34(1): 220-234.

PubMed Abstract | Publisher Full Text

Friston KJ, Parr T, Zeidman P, et al.: Dynamic causal modelling of COVID-19 [version 1; peer review: awaiting peer review]. Wellcome Open Res. 2020a; 5: 89 PubMed Abstract | Publisher Full Text| Free Full Text

Friston KJ, Parr T, Zeidman P, et al.: Second waves, social distancing, and the spread of COVID-19 across America [version 1; peer review: awaiting peer review]. Wellcome Open Res. 2020b; 5: 103.

Publisher Full Text

Friston KJ, Parr T, Zeidman P, et al.: Dynamic Causal Modelling of CovID-19 (Version 4). figshare. 2020c.

http://www.doi.org/10.6084/m9.figshare.12174006.v4

Giordano G, Blanchini F, Bruno R, et al:: Modelling the CovID-19 Epidemic and Implementation of Population-Wide Interventions in Italy. Nat Med. 2020;

1-6.

PubMed Abstract | Publisher Full Text | Free Full Text

Gurdasani $\mathrm{D}$, Ziauddeen $\mathrm{H}$ : On the fallibility of simulation models in informing pandemic responses. Lancet Glob Health. 2020; 8(6): e776-e777. PubMed Abstract | Publisher Full Text | Free Full Text

Hellewell J, Abbott S, Gimma A, et al.: Feasibility of controlling CovID-19 outbreaks by isolation of cases and contacts. Lancet Glob Health. 2020; 8(4): e488-e496.

PubMed Abstract | Publisher Full Text | Free Full Text

Huang CL, Wang YM, Li XW, et al: Clinical features of patients infected with 2019 novel coronavirus in Wuhan, China. Lancet. 2020; 395(10223): 497-506. PubMed Abstract | Publisher Full Text | Free Full Text

Keeling MJ, Hollingsworth TD, Read JM: The Efficacy of Contact Tracing for the Containment of the 2019 Novel Coronavirus (COVID-19). medRxiv. 2020 Publisher Full Text 
Kissler SM, Tedijanto C, Goldstein E, et al.: Projecting the transmission dynamics of SARS-CoV-2 through the postpandemic period. Science. 2020; 368(6493): 860-868.

PubMed Abstract | Publisher Full Text | Free Full Text

Kretzschmar M, Rozhnova G, van Boven M: Isolation and contact tracing can tip the scale to containment of COVID-19 in populations with social distancing. Available at SSRN 3562458. 2020.

Publisher Full Text

Mizumoto K, Chowell G: Estimating Risk for Death from 2019 Novel

Coronavirus Disease, China, January-February 2020. Emerg Infect Dis. 2020; 26(6). 1251-1256.

PubMed Abstract | Publisher Full Text

Penny WD, Stephan KE, Mechelli A, et al.: Comparing dynamic causal models. Neuroimage. 2004; 22(3): 1157-1172

PubMed Abstract | Publisher Full Text

Phipps SJ, Grafton RQ, Kompas T: Estimating the true (population) infection rate for COVID-19: A Backcasting Approach with Monte Carlo Methods. medRxiv. 2020

Publisher Full Text

Russell TW, Hellewell J, Jarvis CI, et al: Estimating the infection and case

fatality ratio for coronavirus disease (COVID-19) using age-adjusted data

from the outbreak on the Diamond Princess cruise ship. Euro surveillance:

bulletin Europeen sur les maladies transmissibles = European communicable

disease bulletin 25. 2020.

Publisher Full Text
Streeck $\mathrm{H}$, Schulte $\mathrm{B}$, Kuemmerer B, et al.: Infection fatality rate of SARS-CoV-2 infection in a German community with a super-spreading event. medRxiv. 2020; 2020.2005.2004.20090076.

Publisher Full Text

Verity R, Okell LC, Dorigatti I, et al.: Estimates of the severity of coronavirus disease 2019: a model-based analysis. Lancet Infect Dis. 2020; 20(6): 669-677. PubMed Abstract | Publisher Full Text | Free Full Text

Vespignani A, Tian H, Dye C, et al.: Modelling CoviD-19. Nature Reviews Physics. 2020; 2: 279-281.

Publisher Full Text

Wang D, Hu B, Hu C, et al:: Clinical Characteristics of 138 Hospitalized Patients With 2019 Novel Coronavirus-Infected Pneumonia in Wuhan,

China. JAMA. 2020; 323(11): 1061-1069.

PubMed Abstract | Publisher Full Text | Free Full Text

Winter AK, Hegde ST: The important role of serology for COVID-19 control. Lancet Infect Dis. 2020; 20(7): 758-759.

PubMed Abstract | Publisher Full Text | Free Full Text

Wölfel R, Corman VM, Guggemos W, et al.: Virological assessment of hospitalized patients with COVID-2019. Nature. 2020. 581(7809): 465-469. PubMed Abstract | Publisher Full Text

Yong SEF, Anderson DE, Wei WE, et al.: Connecting clusters of COVID-19: an epidemiological and serological investigation. Lancet Infect Dis. 2020; 20(7): 809-815.

PubMed Abstract | Publisher Full Text | Free Full Text 


\section{Open Peer Review}

\section{Current Peer Review Status:}

\section{Version 2}

Reviewer Report 12 April 2021

https://doi.org/10.21956/wellcomeopenres.18339.r42617

(C) 2021 Wang L. This is an open access peer review report distributed under the terms of the Creative Commons Attribution License, which permits unrestricted use, distribution, and reproduction in any medium, provided the original work is properly cited.

\section{Lin Wang}

Department of Genetics, University of Cambridge, Cambridge, UK

The authors have addressed my comments. My pleasure to suggest the acceptance.

Competing Interests: No competing interests were disclosed.

Reviewer Expertise: Infectious diseases

I confirm that I have read this submission and believe that I have an appropriate level of expertise to confirm that it is of an acceptable scientific standard.

\section{Version 1}

Reviewer Report 15 October 2020

https://doi.org/10.21956/wellcomeopenres.17553.r40101

(C) 2020 Wang L. This is an open access peer review report distributed under the terms of the Creative Commons Attribution License, which permits unrestricted use, distribution, and reproduction in any medium, provided the original work is properly cited.

\section{Lin Wang}

Department of Genetics, University of Cambridge, Cambridge, UK

In this manuscript, Friston and colleagues extended their established compartmental models to estimate the impact of testing and tracking policies in the UK. I'm a bit confused about their findings and conclusions. Please see below my comments:

1. The authors claimed that "under plausible (graded) relaxations of social distancing, a 
rebound of infections is highly unlikely. The emergence of a second wave depends almost exclusively on the rate at which we lose immunity". However, it is clear that the UK is having the second wave for the period explored in this study. So the model may contain some unrealistic assumptions or parameters.

2. In Table 1, the source of parameters is unknown. Which parameters were obtained from other studies? Which parameters were estimated in this study?

3. How the model was fitted to data? Are you using MCMC? MLE? EM?

4. The model does not include the influence of repeated seeding. For example, the UK has detected lots of introductions of SARS-CoV-2 cases, which is much higher than the assumed number of initial cases in this study. ${ }^{1}$

5. The assumptions on contact patterns are oversimplified. Please account for age-stratified contact patterns. ${ }^{2}$

6. The authors assumed that self-isolated cases could not infect others. This is somehow problematic. For example, if a case was isolated at home, he/she can infect household members.

\section{References}

1. Firth JA, Hellewell J, Klepac $P$, Kissler $S$, et al.: Using a real-world network to model localized COVID-19 control strategies.Nat Med. 26 (10): 1616-1622 PubMed Abstract | Publisher Full Text 2. Pybus O, Rambaut A, du Plessis L, Zarebski A, et al.: Preliminary analysis of SARS-CoV-2 importation and establishment of UK transmission lineages. Reference Source

Is the rationale for developing the new method (or application) clearly explained? Partly

Is the description of the method technically sound? Partly

Are sufficient details provided to allow replication of the method development and its use by others?

Partly

If any results are presented, are all the source data underlying the results available to ensure full reproducibility?

Yes

Are the conclusions about the method and its performance adequately supported by the findings presented in the article?

Partly

Competing Interests: No competing interests were disclosed. 
Reviewer Expertise: Infectious diseases

\section{I confirm that I have read this submission and believe that I have an appropriate level of expertise to state that I do not consider it to be of an acceptable scientific standard, for reasons outlined above.}

Author Response 04 Feb 2021

Adeel Razi, University College London, London, UK

Dear Dr. Wang,

We would like to thank you for the time and effort you have spent reviewing our manuscript. Below are the replies to the comments. We hope these revisions are what you had in mind.

The authors claimed that "under plausible (graded) relaxations of social distancing, a rebound of infections is highly unlikely. The emergence of a second wave depends almost exclusively on the rate at which we lose immunity". However, it is clear that the UK is having the second wave for the period explored in this study. So, the model may contain some unrealistic assumptions or parameters.

Dr Wang raises an excellent point; namely that a rebound may occur in the absence of any material loss in immunity. We have used the dynamic causal model in this report to address the distinction between secondary or rebound waves of infection due to the spread of the virus from the initial population to other populations or communities-and how this is distinguished mechanistically from a second wave due to loss of effective immunity in the first population. A full treatment can be found in the following technical report and op-ed

In Table 1, the source of parameters is unknown. Which parameters were obtained from other studies? Which parameters were estimated in this study?

In Table 1, we provide the list of the model parameters (second column) and their prior densities (their mean and variance in the $3^{\text {rd }}$ and $4^{\text {th }}$ column respectively). These are the model parameters which we infer using observed timeseries data of new cases, deaths and recoveries. In Bayesian inference these priors of the model parameters represent the initial beliefs about them which get updated (i.e., inferred) in the light of the incoming data. These prior values are extracted from previous studies which are given in the Table legend. We have copied these below for your convenience:

"Secondary sources (Huang et al., 2020; Kissler et al., 2020; Mizumoto and Chowell, 2020; Russell et al., 2020; Verity et al., 2020; Wang et al., 2020) and:

https://www.statista.com/chart/21105/number-of-critical-care-beds-per-100000inhabitants/

https://www.gov.uk/guidance/coronavirus-COVID-19-information-for-the-public http://www.imperial.ac.uk/mrc-global-infectious-disease-analysis/COVID-19/

These prior expectations should be read as the effective rates and time constants as they manifest in a real-world setting. For example, a four-day period of contagion is shorter than the period that someone might be infectious (Wölfel et al., 2020) ${ }^{[1]}$, on the (prior) assumption that they will self-isolate, when they realise they could be contagious." 
How was the model fitted to data? Are you using MCMC? MLE? EM?

The data fitting procedure is based on the standard variational (Bayesian) inference procedures (Beal, 2003) under Laplace approximation which is very similar in spirit to the EM algorithm. In our implementations we use Variational Laplace to evaluate model evidence $p(y \mid m)$ and the posterior density over model parameters $p(\theta \mid y, m)$. For details of the procedures used, please see [1] where we applied these procedures to the brain imaging timeseries or [2] for an application to the epidemiological timeseries data.

The EM algorithm can be thought of as a simplified version of variational Bayes, in which the posterior distribution is approximated with the product of two marginals (e.g., over parameters and hyperparameters). The ensuing two variational updates correspond to the $\mathrm{E}$ and the $\mathrm{M}$ steps respectively. The simplification in the EM algorithm is that one assumes the approximate posterior is a point mass in the M-step.

The model does not include the influence of repeated seeding. For example, the UK has detected lots of introductions of SARS-CoV-2 cases, which is much higher than the assumed number of initial cases in this study. 1

Dr Wang is absolutely right this model does not incorporate reseeding. We have now included the following (towards the end of the Conclusion section):

"This DCM does not include the influence of repeated seeding. For example, the UK has detected introductions of SARS-CoV-2 cases, which is much higher than the assumed number of initial cases in this study (Firth et al., 2020). However, for simplicity, we have assumed that the community transmission had, quantitatively, a much greater contribution than reseeding. Having said this, it would be interesting to compare models with and without reseeding to test this assumption formally."

The assumptions on contact patterns are oversimplified. Please account for age-stratified contact patterns. 2

We have now added the reference and our explanation in toward the end of the Conclusion section.

"In this work, the model assumptions on contact patterns are simplified for e.g. agestratified contact patterns are not considered (du Plessis et al., 2020). However, the current extensions of the model with eight stratification and contact matrices have been developed and are available in our open-source code base (DEM_COVID_S.m). Recent work on age stratified models using ONS data with age demographics now underwrites the dashboard ${ }^{8}$ based upon the current DCM (DEM_COVID_UK.m). However, in the current model, we have simply lumped together all age groups-and have focused on heterogeneity in contact rates by conditioning them on different locations in the location factor."

The authors assumed that self-isolated cases could not infect others. This is somehow problematic. For example, if a case was isolated at home, he/she can infect household members.

We are operationally defined isolation as isolation from household members. Conversely, the location state 'home' allows for contacts with household members. In fact, the number of contacts at home is a free parameter of the model. This location state stands in for a lowcontact risk state, in contrast to high contact risk states (e.g., grounded workplaces, public transport, football matches et cetera). 
References:

[1] Friston KJ, Mattout J, Trujillo-Barreto N, Ashburner J, Penny W. Variational free energy and the Laplace approximation. Neuroimage. 2007 Jan 1;34(1):220-34. doi:

10.1016/j.neuroimage.2006.08.035. Epub 2006 Oct 20. PMID: 17055746.

[2] Friston KJ, Parr T, Zeidman P et al. Dynamic causal modelling of COVID-19 [version 2; peer review: 2 approved]. Wellcome Open Res 2020, 5:89 (

https://doi.org/10.12688/wellcomeopenres.15881.2)

Competing Interests: No competing interests were disclosed.

Reviewer Report 05 August 2020

https://doi.org/10.21956/wellcomeopenres.17553.r39476

(C) 2020 Colaneri M. This is an open access peer review report distributed under the terms of the Creative Commons Attribution License, which permits unrestricted use, distribution, and reproduction in any medium, provided the original work is properly cited.

\section{Marta Colaneri}

Division of Infectious Diseases I, Fondazione IRCCS Policlinico San Matteo, Pavia, Italy

This paper presents a thorough analysis of the effects of FTTI (find, track, trace, isolate) in the dynamics of the pandemic in UK. The theoretical development follows one previous paper of the authors where a dynamic causal model of COVID-19 has been proposed. A new state (isolation state) is here added to model the effect of FTTI on the potential occurrence of the second wave of infection as a function of the rate at which immunity is lost in the first wave. A comparison between lethality in UK and Germany is discussed.

I believe that the paper might be useful for political decision-makers in phases 2 and 3. Nevertheless, I ask the authors to comment on the following issues, some of them criticizing the final conclusions:

1. The underlying epidemiological model is quite complex with four macrostates-factors (Infection, Location, Testing, Symptoms), each subdivided in mutually exclusive states. As far as I can understand, the model is a mean-field approximation (to the first moments?) of an "atomic" model of the hiddel Markov chain, with equal individual propensities to change the state.In order to fully understand the temporal evolution (Kolmogorov forward bilinear equation)of the marginal probabilities of the 18 states it would be nice (if space allows) to have the mathematical model displayed along with the transition probability parameters.Does the model incorporate the probability distribution of the pairs in different factors?

2. In the legend of Fig. 1 it is written that one can move from the deceased state to the asymptomatic state, in order to preserve the probability mass (sum=1). Usually, the compartment of deaths is the integral of a linear combination of other states (and hence 
monotonically increasing). Could you please comment further?

3. There are indeed no absorbing states if immunity can be lost. However (as apparent from the simulations) an immunity time of 16 months seems to be large enough to drive the marginal probabilities of the infection status to constant values ("quasi" equilibrium). Could you please comment on the one-step transition probability from resistant to susceptible?

4. In standard epidemiological models (like SEIR)the effective reproduction number is the product between the basic reproduction number and the fraction of susceptible. Stability of the epidemic equilibrium can be characterized easily. I wonder how the theoretical development (of stability and convergence) can be done with time-varying (testing) parameters and Zeno-like behavior of the trajectories if other infection waves occur (in the absense of vaccine or effective therapies).

5. It seems that the resistant status in the infection factor is constant over time, i.e. its probability not affected by other status in other factors. Its value ( 0.38 in UK, 0.58 in DE) seems to be rather high and of course enforce herd immunity (low number of suceptibles left).

6. Could the model respond to the following question: who and where to test at observation time $t+1$ in order to maximize the detection of infected people? This question is motivated by the feeling that the second wave (small in terms of infections, and even before immunity loss) is the summation of very small infection clusters distributed in the territory, and a random network extension of an epidemiological models (endowed with node statistics) is highly welcome.In the same vein, the "return infections" seems to be important after the international border disclosures.

7. Estimation of the number of infectives (in the "hidden" compartment) from noisy data (diagnosized infective, healed, deaths, number of tests etc...) is a real challenge and different approaches are on stage, with very different outcomes (number of total cases from 2 times to 15 times the number of diagnosized cases). How the results of the present paper are settled in this issue?, see e.g.the recent publications:

[*] Boehning at all, "Estimating the undetected infections in the Covid-19 outbreak by harnessing capture-recapture methods", J. Infection Disease, 2020 . $^{1}$

[**] Philipps et al, "Estimating the true (population) infection rate for COVID-19: A Backcasting Approach with Monte Carlo Methods",https://doi.org/10.1101/2020.05.12.20098889.2

I strongly agree with the sentence: this narrative depends on the acquisition of herd immunity .... However, in my view the inferred evidence of immunity in Figure 11 (Streek et $a$ l) is overestimate, being based on data collected in a super spread event. The difference of lethality with Germany is justified by the difference of resistant host of the population, and not on the greater propensity to test and tracing, availability of ICU beds and excellent functioning of the territorial health system, as claimed in a recent guest post by L. Wieler at al, “Emerging COVID-19 success story: Germany's strong enabling environment", June 30, 2020.

\section{References}

1. Böhning D, Rocchetti I, Maruotti A, Holling H: Estimating the undetected infections in the Covid19 outbreak by harnessing capture-recapture methods.Int J Infect Dis. 2020; 97: 197-201 PubMed 


\section{Abstract | Publisher Full Text}

2. Phipps S, Grafton R, Kompas T: Estimating the true (population) infection rate for COVID-19: A Backcasting Approach with Monte Carlo Methods. medRxiv. 2020. Publisher Full Text

Is the rationale for developing the new method (or application) clearly explained? Yes

Is the description of the method technically sound?

Partly

Are sufficient details provided to allow replication of the method development and its use by others?

Yes

If any results are presented, are all the source data underlying the results available to ensure full reproducibility?

Yes

Are the conclusions about the method and its performance adequately supported by the findings presented in the article?

Yes

Competing Interests: No competing interests were disclosed.

Reviewer Expertise: Infectious Diseases

I confirm that I have read this submission and believe that I have an appropriate level of expertise to confirm that it is of an acceptable scientific standard, however I have significant reservations, as outlined above.

Author Response 04 Feb 2021

Adeel Razi, University College London, London, UK

Dear Dr. Colaneri,

We would like to thank you for the time and effort you have spent reviewing our manuscript. Below are the replies to the comments. We hope these revisions are what you had in mind.

The underlying epidemiological model is quite complex with four macrostates-factors (Infection, Location, Testing, Symptoms), each subdivided in mutually exclusive states. As far as I can understand, the model is a mean-field approximation (to the first moments?) of an "atomic" model of the hidden Markov chain, with equal individual propensities to change the state. In order to fully understand the temporal evolution (Kolmogorov forward bilinear equation) of the marginal probabilities of the 18 states it would be nice (if space allows) to have the mathematical model displayed along with the transition probability parameters. Does the model incorporate the probability distribution of the pairs in 


\section{different factors?}

The transition probabilities between states within a factor can indeed depend on the current state in more than one factor. For example, the probability of dying after developing acute respiratory distress syndrome (ARDS) depends on whether one is in a critical care unit (CCU). The transition probabilities are outlined in detail in (Friston et al., 2020a) in the "Transition probabilities and priors" section. Updates for the present paper that incorporate new Infection and Location states are available in the code as described in the software note. We had anticipated that interested readers would refer to the first (foundational) paper in this series for the mathematical details (i.e., the master equation). However, we have added the following (second paragraph in section "Dynamic causal modelling") who just want an intuition as to the basis of the model:

"Although this model may look complicated; it is a straightforward compartmental model that can be written down in terms of a Master Equation, describing the discrete time updates of the joint probability distribution over the four factors. Please see (Friston et al., 2020a). Updating the joint probability (i.e., probability over all tuples of different states) allows us to model transitions among the states of one factor that depend upon other factors."

In the legend of Fig. 1 it is written that one can move from the deceased state to the asymptomatic state, in order to preserve the probability mass (sum=1). Usually, the compartment of deaths is the integral of a linear combination of other states (and hence monotonically increasing). Could you please comment further?

This was just for convenience. In other words, the dwell time in the deceased state can be read directly as the daily fatality rate (reported empirically). Transitions to the susceptible state assume that the number of people dying is balanced by the number of people being born, which is a simplifying but plausible approximation.

There are indeed no absorbing states if immunity can be lost. However (as apparent from the simulations) an immunity time of 16 months seems to be large enough to drive the marginal probabilities of the infection status to constant values ("quasi" equilibrium). Could you please comment on the one-step transition probability from resistant to susceptible?

Although there are no absorbing states, having a very slow transition from the resistant state to the susceptible state can engender a quasi-equilibrium over short timescales. The slow transition from the resistant state to the susceptible state stands in for a loss of cross immunoreactivity, T cell memory - or indeed, an exchange of resistant individuals with another country (with predominantly susceptible individuals).

In standard epidemiological models (like SEIR)the effective reproduction number is the product between the basic reproduction number and the fraction of susceptible. Stability of the epidemic equilibrium can be characterized easily. I wonder how the theoretical development (of stability and convergence) can be done with time-varying (testing) parameters and Zeno-like behavior of the trajectories if other infection waves occur (in the absence of vaccine or effective therapies).

We agree that the stability of and convergence to equilibrium points - under different testing scenarios - are of great interest, and have begun to consider this question in a subsequent manuscript which is currently under peer review: 
https://www.medrxiv.org/content/10.1101/2020.06.11.20128611v1

It seems that the resistant status in the infection factor is constant over time, i.e. its probability not affected by other status in other factors. Its value (0.38 in UK, 0.58 in DE) seems to be rather high and of course enforce herd immunity (low number of susceptible left).

The resistant proportion is estimated from the data and these values, which are by definition higher than those in models that assume that $100 \%$ population susceptibility to a novel virus, offer the best account for the data under the current model structure. In subsequent iterations of the model (one published at https://wellcomeopenresearch.org/articles/5-204/v2 and another under peer review https://www.medrxiv.org/content/10.1101/2020.09.01.20185876v1), we separately consider heterogeneity of exposure, susceptibility, and transmission. This allows for the possibility that a subset of those we define here as "resistant" may still pick up the virus, test positive in PCR tests, but not (so readily) transmit the virus to others. We are also developing the model to allow for changes in the exposed population, for example through population fluxes (https://www.fil.ion.ucl.ac.uk/spm/covid-19/TR5_Second_Wave.pdf and https://www.fil.ion.ucl.ac.uk/spm/covid-19/TR6_DCM.pdf), but this is probably beyond the scope of the current report.

Could the model respond to the following question: who and where to test at observation time $t+1$ in order to maximize the detection of infected people? This question is motivated by the feeling that the second wave (small in terms of infections, and even before immunity loss) is the summation of very small infection clusters distributed in the territory, and a random network extension of an epidemiological models (endowed with node statistics) is highly welcome. In the same vein, the "return infections" seems to be important after the international border disclosures.

Local versions of the model at the level of Local Authorities in England have been implemented, the results of which are viewable at https://fil.ion.ucl.ac.uk/spm/covid19/dashboard/local/ alongside actual test result data. This kind of dashboard could in principle be used to guide decisions on where to focus testing resources to maximize detection of infection. We do not currently model connections between these regions, although we are considering in ongoing work the relative impact of population fluxes ("return infections") versus loss of individual immunity on the development of secondary and second waves of infection.

Estimation of the number of infectives (in the "hidden" compartment) from noisy data (diagnosed infective, healed, deaths, number of tests etc....) is a real challenge and different approaches are on stage, with very different outcomes (number of total cases from 2 times to 15 times the number of diagnosed cases). How are the results of the present paper settled in this issue? See e.g., the recent publications:

$\left[{ }^{*}\right]$ Boehning et al, "Estimating the undetected infections in the Covid-19 outbreak by harnessing capture-recapture methods", J. Infection Disease, 2020.

[**] Philipps et al, "Estimating the true (population) infection rate for COVID-19: A Backcasting Approach with Monte Carlo Methods"

We have now included the references provided by the reviewer and added (in the Conclusion) 
"Here we also note that the estimation of the true number of infected people from noisy timeseries data is a real challenge and different approaches are proposed, with very different outcomes (number of total cases from 2 times to 15 times the number of diagnosed cases), see (Bohning, Rocchetti, Maruotti, \& Holling, 2020; Phipps, Grafton, \& Kompas, 2020). It is indeed the potential to estimate the true number of infected people (and other key latent variables) that led us to adopt and advocate the generative modelling approach of DCM, coupled with variational Bayesian inference that allows us to quantify and account for uncertainty in the case and death data. A validation of these kinds of estimates, with those provided by Edge Health, was recently reported in the Guardian."

I strongly agree with the sentence: this narrative depends on the acquisition of herd immunity .... However, in my view the inferred evidence of immunity in Figure 11 (Streek et al) is overestimate, being based on data collected in a super spread event.

We acknowledge that seroprevalence varies considerably with the nature of the population and events under consideration. Furthermore, seropositive prevalence may not provide the complete picture, especially in the setting of heterogeneity of susceptibility to infection. We have pursued this in subsequent papers testing the notion of seronegative resistance as a potential explanation for differences between countries - much along the lines suggested by Dr. Colaneri.

The difference of lethality with Germany is justified by the difference of resistant host of the population, and not on the greater propensity to test and tracing, availability of ICU beds and excellent functioning of the territorial health system, as claimed in a recent guest post by L. Wieler at al, "Emerging COVID-19 success story: Germany's strong enabling environment", June 30, 2020.

We agree entirely with Dr. Colaneri's observations, which were borne out in subsequent applications of this model to the unfolding trajectories in several European countries.

Further note: $Y$-axes in Fig. 3 are incorrect, should be something like "reproduction rate" and "proportion (\%)"

Thank you for pointing this out. These typos are now corrected.

Competing Interests: No competing interests were disclosed. 